\title{
Isotopic Determination of Uranium in Soil by Laser Induced Breakdown Spectroscopy
}

\author{
George C.-Y. Chan, Inhee Choi ${ }^{\dagger}$, Xianglei Mao ${ }^{*}$, Vassilia Zorba, \\ Oanh P. Lam, David K. Shuh, Richard E. Russo
}

Lawrence Berkeley National Laboratory, Berkeley, CA 94720, USA

*corresponding author, email: x_mao@lbl.gov

${ }^{\dagger}$ present address: Korea Institute of Nuclear Safety, 62 Gwahakro, Yuseong-gu, Daejeon, South Korea 


\section{Abstract}

Laser-induced breakdown spectroscopy (LIBS) operated under ambient pressure has been evaluated for isotopic analysis of uranium in real-world samples such as soil, with $U$ concentrations in the single digit percentage levels. The study addresses the requirements for spectral decomposition of ${ }^{235} \mathrm{U}$ and ${ }^{238} \mathrm{U}$ atomic emission peaks that are only partially resolved. Although non-linear least-square fitting algorithms are typically able to locate the optimal combination of fitting parameters that best describes the experimental spectrum even when all fitting parameters are treated as free independent variables, the analytical results of such an unconstrained free-parameter approach are ambiguous. In this work, five spectral decomposition algorithms were examined, with different known physical properties (e.g., isotopic splitting, hyperfine structure) of the spectral lines sequentially incorporated into the candidate algorithms as constraints. It was found that incorporation of such spectral-line constraints into the decomposition algorithm is essential for the best isotopic analysis. The isotopic abundance of ${ }^{235} \mathrm{U}$ was determined from a simple two-component Lorentzian fit on the U II $424.437 \mathrm{~nm}$ spectral profile. For six replicate measurements, each with only fifteen laser shots, on a soil sample with $\mathrm{U}$ concentration at $1.1 \% \mathrm{w} / \mathrm{w}$, the determined ${ }^{235} \mathrm{U}$ isotopic abundance was $(64.6 \pm 4.8) \%$, and agreed well with the certified value of $64.4 \%$. Another studied U line - U I $682.691 \mathrm{~nm}$ possesses hyperfine structure that is comparatively broad and at a significant fraction as the isotopic shift. Thus, ${ }^{235} \mathrm{U}$ isotopic analysis with this $\mathrm{U}$ I line was performed with spectral decomposition involving individual hyperfine components. For the soil sample with

$1.1 \% \mathrm{w} / \mathrm{w} \mathrm{U}$, the determined ${ }^{235} \mathrm{U}$ isotopic abundance was $(60.9 \pm 2.0) \%$, which exhibited a relative bias about $6 \%$ from the certified value. The bias was attributed to the spectral resolution 
of our measurement system - the measured line width for this U I line was larger than its isotopic splitting. Although not the best emission line for isotopic analysis, this U I emission line is sensitive for element analysis with a detection limit of $500 \mathrm{ppm} U$ in the soil matrix; the detection limit for the U II $424.437 \mathrm{~nm}$ line was $2000 \mathrm{ppm}$.

Keywords: Isotopic analysis; Uranium; Laser induced breakdown spectroscopy; Spectral decomposition; Atomic hyperfine structure 


\section{Introduction}

The ability to characterize uranium isotopic compositions is vital to many nuclear sub-disciplines (e.g., nuclear industry, nuclear forensics, safeguards, and regulatory agencies) [1-3]. For example, the enrichment level of ${ }^{235} \mathrm{U}$ provides insight into useful information about the sample, such as its origin (e.g., nuclear weapons, nuclear power or research reactor fuel, natural uranium, or depleted uranium) [2]. An enrichment level for ${ }^{235} U$ up to $4.5 \%$ generally sets the threshold between peaceful and rogue uses of uranium [4]. Without dispute, mass spectrometry is a powerful method for uranium isotopic analysis. For heavy elements like uranium, the interactions between the electrons and the electric charge distribution of the nucleus [5], which is isotope specific, shift the electronic energies of the atomic levels, and hence the wavelengths of the atomic emission transitions. Isotopic splitting in atomic emission lines provides a physical basis for isotopic analyses with optical atomic spectrometry, which has been demonstrated through several approaches such as atomic absorption [6-8], atomic emission [4, 9-17], atomic fluorescence $[1,18,19]$, and optogalvanic spectroscopy [20, 21].

Photon-emission based measurements offer several unique capabilities over MS-based techniques. First, in contrast to most mass spectrometric techniques, no so-called mass bias correction is needed for $U$ isotopic analysis in emission-based methods [15, 22]. Second, as photons are clean $[23,24]$, there is no radioactive contamination to the main assembly of the instrument - a crucial issue that cannot be avoided using mass spectrometry [22]. Third, photonemission based measurements offer the option to perform standoff or remote analysis [25-27]. 
Since the first demonstration of isotopic analysis of uranium by optical emission spectrometry with an arc discharge as the excitation source [9], many other atomic excitation sources have been developed for isotopic analysis of $\mathrm{U}$, including hollow cathode discharge operated at reduced pressure $[28,29]$, inductively coupled plasma-atomic emission spectrometry (ICPAES) [10-15] conducted at ambient pressure, and laser induced breakdown spectrometry (LIBS) performed either under reduced [16] or at atmospheric pressures $[4,17]$. Despite the fact that the first work on isotopic analysis of uranium utilizing optical atomic emission was published at least six decades ago, such an analytical task is still challenging with current technology because, even for those isotopes that their isotopic shifts are considered to be substantial, the absolute differences in emission wavelengths are nevertheless small. For instance, U II at $424.437 \mathrm{~nm}$ is one of those $\mathrm{U}$ lines that is classified as having a large isotope shift, and has been frequently utilized for $\mathrm{U}$ isotopic analysis $[4,10,12,13,15,16,28-32]$; the absolute magnitude in its ${ }^{235} \mathrm{U}-$ ${ }^{238} \mathrm{U}$ isotope shift is only about $25 \mathrm{pm}$. Although baseline separations for the two isotopic peaks have been reported with ICP-AES operated under atmospheric pressure [11, 13-15], the required instrumentation to obtain such spectral resolution is generally demanding. Data analysis, typically involving simple ratioing of the areas under the individual isotopic peaks $[11,14,15$, 17], is rather straightforward if the two isotopic peaks are baseline resolved. Even with only partially resolved isotopic peaks, accurate isotopic analysis is still feasible with advanced data processing techniques, which has been demonstrated with the partial least square (PLS) calibration method $[4,10]$.

Isotopic analysis of uranium by LIBS potentially offers additional advantages, but also with added challenges, over other atomic excitation sources. Samples are directly analyzed with LIBS without the need to perform laborious sample preparation (e.g., matrix separation). The 
analysis is not only faster, but also results in less radiation dose originating from the sample to laboratory personnel [22]. Moreover, as LIBS can be performed in on-site, in-situ, and standoff fashions, the costs associated with sample collection, transportation, sample preparation, and analysis time are reduced [33]. However, emission-line widths are broadened when LIBS is employed, in particular under atmospheric pressure. The effects from both Doppler and Stark broadening are considerable at atmospheric pressure LIBS [4], and it has been suggested that, with LIBS analysis, the two isotopic spectral profiles can be separated only under reduced pressure conditions [16].

As spectral line emission is transient and varies with time in LIBS, a gated detector is generally necessary to obtain the optimal measured line width (which is particularly important for isotopic analysis), signal-to-background ratio and emission intensity [17, 34]. The choice of a multichannel detector that is able to register emission intensities simultaneously at an array of wavelengths and with temporal gating capability is currently very limited, and most LIBS measurements are performed with an intensified charged coupled device (ICCD). Undesirably, due to electron spreading to adjacent microchannels in the image intensifier tubes [35], the intensifier degrades the attainable spectral resolution [36] and reductions in spectral resolution by factors of 2 to 3 have been reported [37]. Therefore, even though baseline-separated measurements of the ${ }^{235} \mathrm{U}$ and ${ }^{238} \mathrm{U}$ components at U II $424.437 \mathrm{~nm}$ with LIBS under atmospheric pressure has been reported [17], both the hardware requirement and the optimization of operating conditions are challenging [17]. In cases when only partially resolved isotopic $U$ peaks are acquired, isotope ratios still can be extracted from the spectra through the use of chemometrics (e.g., PLS), as successfully demonstrated by Doucet et al. [4] with atmospheric pressure LIBS. 
However, the fact that PLS calibration requires the availability of a series of isotopically enriched standards potentially could be a limitation.

The goal of the present work was to develop an approach for isotopic analysis of uranium with LIBS under atmospheric pressure with relaxed calibration and spectral-resolution requirements. Specifically, the method developed requires no calibration with isotopically enriched standards and the acquired isotopic spectral peaks only need to be partially resolved. Extraction of isotopic information was performed with decomposition of partially resolved spectral peaks. Several spectral decomposition algorithms were examined; different known physical properties (e.g., isotopic splitting, hyperfine structure) of the spectral lines were successively incorporated into the candidate algorithms as constraints. One main objective of the present study was to understand the effect of treating related fitting variables from the two isotopes as independent or linked variables on the isotopic analysis results. Another objective was to evaluate the analytical capability of LIBS for elemental and isotopic analysis of uranium in an environmental sample matrix. The sample employed in this study was U-doped soil, with U concentrations in the single percentage levels.

\section{Experimental}

\subsection{Sample and sample preparation}

Isotopically enriched $\mathrm{U}_{3} \mathrm{O}_{8}$ powders were blended with soil to give a final $\mathrm{U}$ concentration in the single digit percentage level. Certified enriched $\left(63 \%{ }^{235} \mathrm{U}\right.$, CRM U630) $\mathrm{U}_{3} \mathrm{O}_{8}$ powder was obtained from New Brunswick Laboratory of the U.S. Department of Energy. Neglecting the 
trace amount of ${ }^{234} \mathrm{U}$ and ${ }^{236} \mathrm{U}$ in CRM U630, the atom fraction of ${ }^{235} \mathrm{U} /\left({ }^{235} \mathrm{U}+{ }^{238} \mathrm{U}\right)$ is $64.37 \%$. The soil matrix that was used in this study was a standard reference material (SRM 2710a) from NIST. This soil SRM is described as Montana I soil and its major (>0.5\% w/w) elemental compositions are Si (31.1\%), Al (5.95\%), Fe (4.32\%), K (2.17\%), Ca (0.964\%), Na (0.894\%), $\mathrm{Mg}(0.734 \%)$, and $\mathrm{Pb}(0.552 \%)$. Although a trace amount of $\mathrm{U}$ is also present in the soil matrix, the amount is negligible and both the $\mathrm{U}$ concentration and its isotopic composition should be defined by the amount of $\mathrm{U}_{3} \mathrm{O}_{8}$ that was added. The certified mass fraction of $U$ in SRM 2710a is $9.11 \mathrm{mg} / \mathrm{kg}$, and is three orders of magnitude less than the lowest $\mathrm{U}$ concentration $(1.1 \% \mathrm{w} / \mathrm{w})$ used in the present study.

Four portions of CRM U630 $\mathrm{U}_{3} \mathrm{O}_{8}$ powder, with masses 1.3, 3.0, 5.0, and $10.2 \mathrm{mg}$ were weighed and blended with 99, 97, 95, and $90 \mathrm{mg}$ of the SRM 2710a soil matrix, respectively. The concentrations of uranium in the blends were $1.1,2.5,4.2$ and $8.6 \%$ w/w. A blank, which consisted of $100 \mathrm{mg}$ of SRM 2710a, was also prepared. These powered samples were individually placed into a pellet-pressing die with a diameter of $3 \mathrm{~mm}$, and forces of approximately 6 tons were then applied for 3 minutes. The prepared U-doped soil sample pellets were about $1 \mathrm{~mm}$ thick. The sample pellets were then loaded into a shielded chamber, which contained air at atmospheric pressure, with optical ports for the laser ablation and photon collection.

\subsection{LIBS system and measurements}

The experimental setup was similar to our previous studies $[34,38]$ and consisted of a Nd:YAG laser, a 1.25 m-focal length Czerny-Turner spectrometer, and an ICCD gated detector. Briefly, 
the Nd:YAG laser was operated at its fundamental wavelength at $1064 \mathrm{~nm}$ with a pulse duration about 5 ns. Laser energy was approximately $40 \mathrm{~mJ} /$ pulse. The measured pulse-to-pulse fluctuations in laser power were only 1\% RSD. The laser beam was focused onto the soil-pellet sample surface, which was placed inside a sealed chamber, through the designated optical port. The diameter of the laser spot on the sample was approximately $350 \mu \mathrm{m}$. Plasma emission was collected orthogonal to the incoming laser-beam direction, through another optical port, with a second fused-silica lens. The laser-induced plasma emission was then directed to the entrance slit of the spectrometer through an optical fiber bundle. The plasma emission was registered by an ICCD detector with typical operating parameters as in other LIBS measurements - a delay time of $1.5 \mu$ s and a gate width of $20 \mu$ s. Each measurement consisted of an accumulation of emission signals from 15 laser shots on a single fixed spot on the sample, and six replicate measurements were made on each sample.

Two uranium emission lines - U I $682.691 \mathrm{~nm}$ and U II $424.437 \mathrm{~nm}$ were measured in this study. Herein, the quoted wavelength of an emission line always refers to that emitted from ${ }^{238} \mathrm{U}$, even during the discussion of ${ }^{235} \mathrm{U}$ emission. These two lines were chosen because our previous study [38], in which a pool of forty-three atomic emission lines of $U$ were simulated for their analytical performance for isotopic analysis, showed that they are the two best lines for isotopic analysis of uranium. Although the isotopic splitting of the U I $682.691 \mathrm{~nm}$ line is only moderate $(17.7 \mathrm{pm})$, it is the strongest LIBS line for uranium [38, 39], and one identified primary factor limiting the precision of LIBS isotopic analysis is photon-shot noise level [38]. The U II $424.437 \mathrm{~nm}$ line is the second strongest line measured with our LIBS system, and it possess a larger isotopic splitting $(25.1 \mathrm{pm})$. These two $\mathrm{U}$ lines were measured with different gratings. The U I line was measured with a grating with groove density 2400 per mm, which gives a measured spectral 
bandpass (expressed as full width at half maximum, FWHM) about $18 \mathrm{pm}$. A higher groovedensity grating with 3600 per mm, which offers a spectral bandpass of $12 \mathrm{pm}$, was used for the measurements of the U II line.

\section{Results and Discussion}

\subsection{U spectra and candidate algorithms for isotopic spectral decomposition}

Figures 1a and $1 \mathrm{~b}$ show the LIBS U spectra at $682.691 \mathrm{~nm}$ and $424.437 \mathrm{~nm}$, respectively, obtained at the lowest studied $\mathrm{U}$ concentration $(1.1 \% \mathrm{w} / \mathrm{w})$ and the soil blank. The two isotopic components $\left({ }^{235} \mathrm{U}\right.$ and $\left.{ }^{238} \mathrm{U}\right)$ appeared with significant overlap for the U I $682.691 \mathrm{~nm}$ line whereas, although unresolved to the baseline, they are adequately resolved at U II $424.437 \mathrm{~nm}$. The better resolution for the two isotopic components for U II $424.437 \mathrm{~nm}$ is attributed to several factors: a larger isotopic splitting, the use of a higher groove-density grating, and negligible hyperfine structure (see next section for further discussion). Although not baseline resolved, it is still possible to extract isotopic composition of the sample from such spectra. Our previous study [34], with a U-containing sample at natural isotopic abundance, confirmed that the measured U lines are well represented by Lorentzian profiles for two reasons. First, as it has been reported that the microchannel-plate intensifier could degrade the spectral resolution of a spectrometer by factors as large as 3 [36, 37], the instrumental profile of the whole spectrometric-detector system is largely dictated by the intensifier of the ICCD. Instrumental profile of intensifier, which can be obtained through inverse Fourier transform of its modulation transfer function (MTF), is generally Lorentzian like [40]. The instrumental profile of our spectrometric (spectrometer-ICCD detector) system was verified by measurement of the $\mathrm{Hg}$ line 
at $435.833 \mathrm{~nm}$ emitted from a low-pressure $\mathrm{Hg}$ pen lamp, and it was found that the instrumental profile can be well represented by a Lorentzian function. Second, Stark broadening, which gives Lorentzian line profile, is considered the dominant line-broadening mechanism [41, 42] in atmospheric-pressure LIBS. Therefore, the spectral profiles of ${ }^{235} \mathrm{U}$ and ${ }^{238} \mathrm{U}$ are assumed to be Lorentzian in the present study.

A non-linear least-square fitting algorithm, written in house with LabVIEW (National Instruments, Austin, TX, USA) software, was applied to the experimental spectra. The fitting function (Equation 1) is a linear sum of four terms with the first and second terms representing the atomic emission from ${ }^{235} \mathrm{U}$ and ${ }^{238} \mathrm{U}$, respectively. The third term denotes the plasma continuum background, which is assumed to vary linearly with wavelength within the small spectral window (typically $~ 200$ to $300 \mathrm{pm}$ ) employed in the fitting. The last term in Equation 1 represents the residual of the fitting. The fitting algorithm locates the best combination of all fitting parameters by minimization of the sum of the squares of the fitting residuals $(\varepsilon(\lambda))$ at all wavelengths $(\lambda)$ of the experimental spectrum, $I^{\text {expt }}(\lambda)$. Altogether there are eight fitting variables: for the two isotopic components, $A, \omega$ and $\lambda^{\circ}$ denote the peak area, Lorentzian width and the center wavelength of the atomic emission line with subscripts 235 and 238 referring to the specific isotopic components. The decomposed profiles from the two isotopes depicted in Figure 1 illustrate these fitting parameters for the atomic line. Because wavelength calibration of the spectrometer was not perfect and could be subjected to shift and drift, the center emission wavelengths are included as fitting parameters to correct for the slight wavelength bias in the wavelength calibration. For the plasma continuum, $c_{1}$ and $c_{2}$ represent the constant and linearvarying portions of the background. 


$$
I^{\operatorname{expt}}(\lambda)=\frac{A_{235} \omega_{235}}{2 \pi\left[\left(\lambda-\lambda_{235}^{\mathrm{o}}\right)^{2}+\left(\frac{1}{2} \omega_{235}\right)^{2}\right]}+\frac{A_{238} \omega_{238}}{2 \pi\left[\left(\lambda-\lambda_{238}^{\mathrm{o}}\right)^{2}+\left(\frac{1}{2} \omega_{238}\right)^{2}\right]}+\left[c_{1}+c_{2} \lambda\right]+\varepsilon(\lambda)
$$

Out of the three paired fitting parameters $\left(A, \omega\right.$ and $\left.\lambda^{\circ}\right)$ for the two isotopic components, only $A_{235}$ and $A_{238}$ are completely unrelated to each other and depend only on the concentrations of ${ }^{235} \mathrm{U}$ and ${ }^{238} \mathrm{U}$ in the sample, respectively. In contrast, $\lambda_{235}^{\mathrm{o}}$ and $\lambda_{238}^{\mathrm{o}}$ are related to the isotopic splitting (IS) of the emission line, which are well documented for both U I $682.691 \mathrm{~nm}$ and U II $424.437 \mathrm{~nm}$. Likewise, $\omega_{235}$ and $\omega_{238}$ are correlated because the line-broadening mechanisms apply similarly to both isotopic components. As mentioned in the introduction, an objective of the present study is to understand the effect of treating related fitting variable as independent or linked variables in the decomposition algorithm. Therefore, the results from five fitting algorithms, which are summarized in Table 1, were compared with increasing complexity in the constraints. In all these algorithms, the determined atom fraction of ${ }^{235} U /\left({ }^{235} U+{ }^{238} U\right)$ in the sample is deduced directly from the ratios of $A_{235} /\left(A_{235}+A_{238}\right)$ after spectral decomposition.

In Algorithm I, no constraint is added $-\lambda_{235}^{0}, \lambda_{238}^{0}, \omega_{235}$, and $\omega_{238}$ are all treated as free fitting variables. In Algorithm II, the wavelength differences between the centres of the two isotopic peaks are set to the published isotopic shifts. Algorithm III is based on Algorithm II with an additional constraint that the peak widths of the ${ }^{235} \mathrm{U}$ and ${ }^{238} \mathrm{U}$ components are identical; this constrain neglects the hyperfine structure of ${ }^{235} \mathrm{U}$ and assumes that identical line-broadening effects apply to both isotopic components. Algorithm IV is similar to Algorithm III but takes into the account of hyperfine structure of ${ }^{235} \mathrm{U}$ and assumes that the sum of all hyperfine structure of ${ }^{235} \mathrm{U}$ can still be well represented by a single Lorentzian profile with a slightly larger width. The constant $\boldsymbol{k}$ linking the line widths of ${ }^{235} \mathrm{U}$ and ${ }^{238} \mathrm{U}$ is not a fitting variable; rather, it is 
predefined from theoretical calculation based on the measured line width of ${ }^{238} \mathrm{U}$. The calculation and their relations with ${ }^{238} \mathrm{U}$ line widths will be presented in Section 3.2. Algorithm $\mathrm{V}$ is the most elaborate fitting algorithm; the ${ }^{235} \mathrm{U}$ spectral profile is no longer treated as a single Lorentzian profile. The hyperfine shift and emission strength for each hyperfine component is first calculated and then fitted to the experimental spectra. Calculation of hyperfine pattern is based on published hyperfine constants and theoretical intensity distribution, and will be discussed in detail in Section 3.2. In Algorithm V, the fitting function for ${ }^{235} \mathrm{U}$ (i.e., the first term in Equation 1) is modified to

$$
I_{235 U}(\lambda)=\frac{A_{235}}{2 \pi} \sum_{\forall i} \frac{f_{H F S-i} \cdot \omega_{235}}{\left[\left(\lambda-\lambda_{235, H F S-i}^{o}\right)^{2}+\left(\frac{1}{2} \omega_{235}\right)^{2}\right]}
$$

where $\lambda_{235, H F S-i}^{o}$ denotes the centre emission wavelength of the $i^{\text {th }}$ hyperfine structure (HFS) component with fractional emission strength $f_{H F S-i}\left(\right.$ i.e., $\Sigma f_{H F S-i}=1$ ); the spectral profile for each hyperfine component is assumed to be Lorentzian with identical width.

\subsection{Hyperfine structure and isotopic shift of ${ }^{235} \mathrm{U}$ emission lines}

Hyperfine structure in the atomic spectra of ${ }^{235} \mathrm{U}$ results from the coupling of electron angular momentum $(\boldsymbol{J})$ with the nuclear spin $\left(\boldsymbol{I}=7 / 2\right.$ for $\left.{ }^{235} \mathrm{U}\right)$. In contrast, HFS is absent in ${ }^{238} \mathrm{U}$ as its nucleus possesses no nuclear $\operatorname{spin}\left(I=0\right.$ for $\left.{ }^{238} U\right)$. The incomplete filling of the $f$ - and $d$-shells of uranium atom/ion result in many electronic configurations with large electron angular momentum. When this large electron angular momentum couples with a similarly large nuclear angular momentum, the total angular momentum $(\boldsymbol{F}=\boldsymbol{J}+\boldsymbol{I})$ is even larger. For instance, both 
the upper and lower energy levels of the U I $682.691 \mathrm{~nm}$ transition have $J=6$, which after coupling with the nuclear spin, split into eight levels each with a different total angular momentum $(\boldsymbol{F}=19 / 2,17 / 2, \ldots 5 / 2)$. In general, the hyperfine structure for many ${ }^{235} \mathrm{U}$ electronic transitions are complex with hyperfine splitting comparable to the isotopic splitting between ${ }^{235} \mathrm{U}$ and ${ }^{238} \mathrm{U}$ in some cases [43, 44], which then further complicates the extraction of isotopic information from the spectra. In this section, the theoretical hyperfine patterns for both the U I 682.691 nm and U II $424.437 \mathrm{~nm}$ lines are calculated, and the effect of HFS on decomposition of isotopic components in the atomic spectra is discussed. Throughout this paper, the quoted wavelength of an emission line refers to that emitted from ${ }^{238} \mathrm{U}$.

Table 2 lists the spectroscopic constants [45-47], ${ }^{235} \mathrm{U}$ isotopic shift $[46,47]$ from ${ }^{238} \mathrm{U}$, and experimentally measured ${ }^{235} \mathrm{U}$ hyperfine constants $[43,48,49]$ for the U I $682.691 \mathrm{~nm}$ and U II $424.437 \mathrm{~nm}$ lines. The two hyperfine constants, $A$ and $B$, denote the nuclear-electronic interactions between magnetic dipole and electric quadrupole, respectively [49-51]. As a first step to compute the hyperfine structure, energy shifts for all the individual hyperfine components in both the upper and lower energy levels of the electronic transition were calculated. Energy shift for HFS $\left(\Delta E_{F}\right)$ can be readily evaluated with the Casimir formula [51, 52]:

$$
\Delta E_{F}=\frac{A C}{2}+\frac{3 B C(C+1)-4 I(I+1) J(J+1)}{8 I(2 I-1) J(2 J-1)}
$$

where $A$ and $B$ are HFS constants, and $I, J$ and $F$ are the nuclear, total electronic and total atomic angular momentum, respectively, and $C$ is defined as 


$$
C=F(F+1)-J(J+1)-I(I+1)
$$

Although each energy level is split into multiple hyperfine levels, the selection rule $(\Delta F=0, \pm 1$, with $F=0 \leftrightarrow F=0$ is forbidden) limits the number of allowed transitions within the HFS. With the above U I $682.691 \mathrm{~nm}$ emission line as an example, despite both the upper and lower energy levels of the transition being split into eight hyperfine levels, there are only 22 allowed components in its HFS. After identification of the allowed components in the HFS, their relative intensities can be computed analogous to relative strengths within a $L S$-coupling multiplet [50, 53]. Table 3 summarizes the hyperfine energy shift (relative to the centroid of all HFS components), percentage relative emission strength and air wavelength of individual HFS component for ${ }^{235} \mathrm{U}$ at U I $682.691 \mathrm{~nm}$. Data for ${ }^{235} \mathrm{U}$ at U II $424.437 \mathrm{~nm}$ are tabulated in Table 4.

For the U I $682.691 \mathrm{~nm}$ line, the principal hyperfine pattern is found with $\Delta F=0$ (cf. Table 3). The span of energy shifts among these eight hyperfine components with $\Delta F=0$ is $147 \mathrm{mK}$, which corresponds to a 6.8-pm shift in wavelength and is a significant fraction compared to the $380 \mathrm{mK}$ isotopic shift, which translates to $17.7 \mathrm{pm}$ in wavelength. The relatively broad hyperfine pattern for U I $682.691 \mathrm{~nm}$ implies that the overall ${ }^{235} \mathrm{U}$ emission profile is broader than the ${ }^{238} \mathrm{U}$ profile and might even be distorted from a single Lorentzian profile. To further understand the effect of hyperfine structure on spectral decomposition from the two uranium isotopes, the emission spectral profiles from a $50 \%-50 \%{ }^{235} \mathrm{U}-{ }^{238} \mathrm{U}$ sample was simulated and depicted in Figure 2a. The simulation assumes that each component is Lorentzian in shape with a 20-pm width; this 20-pm width is similar to the observed line width in the present study. Because the ${ }^{235} \mathrm{U}:{ }^{238} \mathrm{U}$ ratio is $1: 1$ in the simulation and thus the areas under the two isotopic 
peaks area are identical, any difference in their peak heights implies that the peak widths of the two profiles are dissimilar. Figure 2a clearly demonstrates that there is a noticeable difference in the peak heights of the ${ }^{235} \mathrm{U}$ and ${ }^{238} \mathrm{U}$ profiles. Also shown in Figure 2a are the eight principal hyperfine components and it is clear that the center of these HFS components shift from each other, causing an overall broadened line profile. Figure $2 b$ shows the ratios of the theoretical peak widths of ${ }^{235} \mathrm{U}$ to ${ }^{238} \mathrm{U}$, if the ${ }^{235} \mathrm{U}$ profile is to be represented by a single Lorentzian profile, as a function of the ${ }^{238} \mathrm{U}$ line width. These ratios are obtained from fitting a single Lorentzian function to the overall ${ }^{235} \mathrm{U}$ line profile. The ratios are large for narrow line widths and approach the asymptotic value of 1.000 only at large widths. For example, the ratios are 1.183, 1.053 and 1.015 for widths 10, 20 and $40 \mathrm{pm}$, respectively. Thus, it is necessary to correct for the unequal line widths between the overall ${ }^{235} \mathrm{U}$ and ${ }^{238} \mathrm{U}$ spectral profiles, if isotopic analysis is to be performed with a simple two-component Lorentzian fitting of the measured spectrum. Figure $2 \mathrm{~b}$ provides a guideline for setting the value of $\boldsymbol{k}$ in Algorithm IV (cf. Table 1). In our present experiment, the measured line width for the ${ }^{238} \mathrm{U}$ at U I $682.691 \mathrm{~nm}$ is $19.3 \mathrm{pm}$, therefore, $\boldsymbol{k}$ was set to 1.05 for Algorithm IV.

The hyperfine splitting for the U II $424.437 \mathrm{~nm}$ line is small and the overall ${ }^{235} \mathrm{U}$ spectral line is much better represented by a single Lorentzian profile with width similar to the ${ }^{238} \mathrm{U}$ component. The HFS data summarizing the hyperfine energy shift, percentage relative emission strength and air wavelength of individual HFS component for ${ }^{235} \mathrm{U}$ at U II $424.437 \mathrm{~nm}$ is tabulated in Table 4. The span of energy shifts within the eight principal hyperfine components with $\Delta F=+1$ is only $70 \mathrm{mK}$, which translates to wavelength shift of only $1.3 \mathrm{pm}$ at $424.437 \mathrm{~nm}$. Such a span is minor compared to the magnitude of the ${ }^{235} \mathrm{U}-{ }^{238} \mathrm{U}$ isotope shift (1395 mK, which corresponds to $25.1 \mathrm{pm}$, cf. Table 2). Figure 3a shows the simulated spectral profiles from a $50 \%-50 \%{ }^{235} \mathrm{U}-$ 
${ }^{238} \mathrm{U}$ sample. Compared to Figure 2a, the line width in the simulation presented in Figure 3a is reduced by half to only $10 \mathrm{pm}$. Even with a 10-pm line width, the peak heights of the sum of all the ${ }^{235} \mathrm{U}$ HFS components and the ${ }^{238} \mathrm{U}$ peak are similar to within $1 \%$. Figure 3 a also shows that the centers of the eight principal hyperfine components align comparatively close to each other, making it possible to approximate the overall ${ }^{235} \mathrm{U}$ profile as a single Lorentzian profile with a matching width as the ${ }^{238} \mathrm{U}$ profile. The ratios of the theoretical peak widths of ${ }^{235} \mathrm{U}$ to ${ }^{238} \mathrm{U}$ as a function of the ${ }^{238} \mathrm{U}$ line width are presented in Figure 3b. Compared to the U I $682.691 \mathrm{~nm}$ (cf. Figure 2b), the ratios for U II $424.437 \mathrm{~nm}$ approach the 1.00 asymptotic value at widths as narrow as $12 \mathrm{pm}$. As the measured line width for ${ }^{238} \mathrm{U}$ at $\mathrm{U}$ II $424.437 \mathrm{~nm}$ is $14.5 \mathrm{pm}$ in our present experiment, $\boldsymbol{k}$ is nearly unity and Algorithm IV reduces to Algorithm III.

\subsection{Spectral decomposition for ${ }^{235} \mathrm{U}$ and ${ }^{238} \mathrm{U}$ isotopic analysis - a comparison of different algorithms}

Figure 4a shows the determined ratios of ${ }^{235} \mathrm{U} /\left({ }^{235} \mathrm{U}+{ }^{238} \mathrm{U}\right)$ using the U I $682.691 \mathrm{~nm}$ line, as a function of uranium concentration in soil. As different spectral-decomposition constraints (cf. Table 1) were applied to the same set of experimental spectra, the results are directly comparable. The error bars represent the standard deviations of six replicates, each measured with 15 laser shots on a fixed sample spot. Although the determined ratios are algorithm dependent, the ratios are independent of uranium concentrations, suggesting that the biases are caused by the spectral-decomposition algorithms instead of the signal strengths. Without any constraint (i.e., Algorithm I), the determined isotopic ratios express the largest bias; the average determined ${ }^{235} \mathrm{U} /\left({ }^{235} \mathrm{U}+{ }^{238} \mathrm{U}\right)$ ratios from Algorithm I was only $43.2 \%$ and compared unsatisfactorily with the certified value $64.37 \%$ for CRM 630. Algorithm II, which fixes the 
difference in the peak centers of the two fitted isotopic profiles, slightly improved the result to 46.8\%. Noticeable improvements, in terms of both accuracy and precision, were found when the line widths $(\omega)$ of the ${ }^{235} \mathrm{U}$ and ${ }^{238} \mathrm{U}$ were set to be correlated (i.e., Algorithm III and IV). When $\omega_{235}$ is set to be equal to $\omega_{238}$ (i.e., Algorithm III), the averaged isotopic ratios improved to $56.2 \%$ and further improved to $57.6 \%$ when the broadened profile from ${ }^{235} \mathrm{U}$ HFS was taken into account (i.e., Algorithm IV, with $\boldsymbol{k}=1.05$ ). The ${ }^{235} \mathrm{U}$ HFS not only broadens the apparent line width of the ${ }^{235} \mathrm{U}$ profile, but could also distort its shape. Algorithm $\mathrm{V}$, which fits each individual HFS for ${ }^{235} \mathrm{U}$, yielded a determined isotopic ratio of $59.9 \%$, which is closest to the certified value of $64.37 \%$, among the five studied algorithms. An application of a two-sided $t$-test at $95 \%$ confidence concluded that the determined isotopic ratios from Algorithm V were statistically different from the certified value (i.e., bias was present).

A major reason for the biased results from Algorithm $\mathrm{V}$ is believed to stem from the larger-thanone ratio of the measured line width $(\sim 19.3 \mathrm{pm})$ and the isotopic splitting $(\sim 17.7 \mathrm{pm})$. We previously [34] investigated the measured spectral resolution on the accuracy of spectral decomposition. In that study, a doublet from a $\mathrm{Hg}$ emission line with $\Delta \lambda=29 \mathrm{pm}$ was measured under different spectral bandpass, decomposed with two-component spectral fitting, and the ratios of the doublet were then evaluated [34]. When the measured line widths were about $0.5 \times$ (i.e., narrower than) the splitting of the doublet, the area ratios of the decomposed doublet were within $1 \%$ of the theoretical value [34]. However, the accuracies quickly degraded with increasing line widths to $\sim 6 \%$ and $\sim 7 \%$ when the widths were raised to about $1.0 \times$ and $1.5 \times$, respectively, the splitting of the doublet [34]. In the present study, the measured width at U I $682.691 \mathrm{~nm}$ is larger than the isotopic splitting, with a ratio $\sim 1.09$. The averaged experimentally 
determined ${ }^{235} \mathrm{U}$ isotopic ratio from Algorithm $\mathrm{V}$ was $59.9 \%$, and exhibited a $7 \%$ relative bias from the $64.37 \%$ certified value. The bias agreed well with what was expected from the previous Hg-doublet study [34].

The determined ratios of ${ }^{235} \mathrm{U} /\left({ }^{235} \mathrm{U}+{ }^{238} \mathrm{U}\right)$ from the U II $424.437 \mathrm{~nm}$ line are presented in Figure 4b. Compared to the results obtained from the U I $682.691 \mathrm{~nm}$ line (cf. Figure 4a), the analytical performance for ${ }^{235} \mathrm{U}^{238} \mathrm{U}$ isotopic analysis with this U II line is superior. For Algorithms I and II, which respectively contain zero and only one constraint on the isotopic splitting, the averaged determined ${ }^{235} \mathrm{U} /\left({ }^{235} \mathrm{U}+{ }^{238} \mathrm{U}\right)$ ratios were $69.1 \%$ and $70.2 \%$ - comparing satisfactorily with the certified value of $64.37 \%$. The averaged isotopic ratio improved to $64.9 \%$ once an additional constraint for equal line width was added (i.e., Algorithm III). According to the two-sided $t$-test at $95 \%$ confidence level, results from Algorithm III is not statistically different from the certified value. Further, paired $t$-tests ( 2 sided, $95 \%$ confidence) indicate that the determined isotopic ratios from Algorithm III and V (the most elaborated model involving individual HFS components) are statistically identical. Therefore, isotopic analysis of U could be readily determined with a simple two-component spectral decomposition, with a well-defined isotopic splitting constraint and only one spectral-width as a fitting parameter for both ${ }^{235} \mathrm{U}$ and ${ }^{238} \mathrm{U}$. It is also noted that precision improved as $\mathrm{U}$ concentration increased (stronger $\mathrm{U}$ emission). As previously discussed, Algorithm IV reduces to Algorithm III for this U II emission line. As the overall ${ }^{235} \mathrm{U}$ spectral profile can be well represented as a single Lorentzian peak (cf. Figures 3a and 3b), fitting individual ${ }^{235}$ U HFS component (i.e., Algorithm V) offers no further improvement. 


\subsection{Estimation of detection limits of uranium in soil matrix by LIBS}

Figures $5 \mathrm{a}$ and $5 \mathrm{~b}$ show the dependence of the sum of decomposed ${ }^{235} \mathrm{U}$ and ${ }^{238} \mathrm{U}$ signals as a function of uranium concentrations in soil for the emission lines U I $682.691 \mathrm{~nm}$ and U II $424.437 \mathrm{~nm}$, respectively. The relatively large error bars were likely due to inhomogeneous mixing of the minute amount of $\mathrm{U}_{3} \mathrm{O}_{8}$ and soil powders. Nevertheless, both calibration curves express good linearity. Because uranium is practically absent in the blank soil matrix, $A_{235}$ and $A_{238}$, as well as the plasma-continuum parameters, were the only fitting variables for the evaluation of the blank signals; other parameters (e.g., line width, spectral position) were all fixed parameters and adopted from the averages of the fitting results from those U-spiked soil samples. The calculated detection limits were independent of the employed algorithm for the spectral decomposition, and were 500 and 2000 ppm for U I $682.691 \mathrm{~nm}$ and U II $424.437 \mathrm{~nm}$, respectively. Chinni et al. [39] investigated elemental LIBS analysis of uranium in soil under ambient pressure and reported detection limits range from 260 to $1700 \mathrm{ppm}$, depending on the emission lines used. Although none of the two $U$ lines employed in this work were included in the six lines studied by Chinni et al. [39], the detection limits of the two studies are within the same order.

\section{Conclusion}

Isotopic analysis of uranium in spiked soil samples by LIBS operated under atmospheric pressure was demonstrated in this work. The measured atomic emission lines from the two $\mathrm{U}$ isotopes $-{ }^{235} \mathrm{U}$ and ${ }^{238} \mathrm{U}$ were only partially resolved, yet correct isotopic information can be extracted from the spectra through spectral decomposition. Two important factors are identified 
for the success of spectral decomposition for accurate isotopic analysis. First, known physical properties (e.g., isotopic splitting, hyperfine structure) of the spectral lines must be incorporated into the algorithms as constants. Second, the measured line widths (including both physical and instrumental broadenings) should be narrower than the isotopic shifts.

Although non-linear least-square fitting algorithms typically can locate the combination of fitting parameters that best describes the experimental spectrum even when all fitting parameters are utilized as free independent variables, the analytical results of such an all-free-parameter approach are ambiguous. In addition, hyperfine structure could broaden the width and distort the overall ${ }^{235} \mathrm{U}$ emission profile from a single Lorentzian profile. Also, the total wavelength spans of some ${ }^{235} \mathrm{U}$ hyperfine structures are of comparable magnitude as the ${ }^{235} \mathrm{U}-{ }^{238} \mathrm{U}$ isotopic splitting, which further complicate spectral decomposition. Therefore, a detailed analysis of HFS is essential prior to isotopic analysis by optical emission spectrometry. The hyperfine splitting for the U II $424.437 \mathrm{~nm}$ line is negligibly small and the ${ }^{235} \mathrm{U}$ component can be treated as a single Lorentzian profile with an identical width as the ${ }^{238} \mathrm{U}$ component. However, the hyperfine splitting in the U I $682.691 \mathrm{~nm}$ line is large and the overall spectral profile of the ${ }^{235} \mathrm{U}$ component is broadened and distorted from a single line profile. Although not the best emission line for uranium isotopic analysis, the U I $682.691 \mathrm{~nm}$ line is well suited for total uranium determination as it is the strongest $\mathrm{U}$ emission line measured by LIBS; the detection limit, in the soil matrix, was found to be $500 \mathrm{ppm}$ U. Isotopic analysis can be readily performed with a simple two-component spectral decomposition on the U II $424.437 \mathrm{~nm}$ spectral profile. The determined ${ }^{235} \mathrm{U}$ isotopic abundance agreed with the certified value for total uranium concentration at $1.1 \% \mathrm{w} / \mathrm{w}$ in the soil matrix. 


\section{Acknowledgment}

This work was supported by the Defense Nuclear Nonproliferation Research and Development Office (GCC, XLM, IC, VZ, RER), and the Director, Office of Science, Office of Basic Energy Sciences, Division of Chemical Sciences, Geosciences, and Biosciences, Separations and Analysis Program (GCC, XLM, IC, VZ, RER) and the Heavy Element Chemistry Program (OPL, DKS) of the U.S. Department of Energy under contract number DE-AC02-05CH11231 at the Lawrence Berkeley National Laboratory. 


\section{References}

1. J.A. Vera, G.M. Murray, S.J. Weeks, M.C. Edelson, Isotopic abundance determination by inductively coupled plasma high-resolution laser-excited atomic and ionic fluorescence spectroscopy, Spectrochim. Acta Part B 46 (1991) 1689-1700.

2. S. Horne, S. Landsberger, B. Dickson, Determination of isotopic ratios of uranium samples using passive gamma spectroscopy with multiple detectors, J. Radioanal. Nucl. Chem. 299 (2014) 1171-1175.

3. R.C. Marin, J.E.S. Sarkis, M.R.L. Nascimento, The use of LA-SF-ICP-MS for nuclear forensics purposes: Uranium isotope ratio analysis, J. Radioanal. Nucl. Chem. 295 (2013) 99-104.

4. F.R. Doucet, G. Lithgow, R. Kosierb, P. Bouchard, M. Sabsabi, Determination of isotope ratios using laser-induced breakdown spectroscopy in ambient air at atmospheric pressure for nuclear forensics, J. Anal. At. Spectrom. 26 (2011) 536-541.

5. W.H. King, Isotopic shifts in atomic spectra, $1^{\text {st }}$ ed., Plenum Press, New York (1984).

6. J.A. Goleb, Determination of uranium isotopes by atomic absorption spectrophotometry, Anal. Chim. Acta 34 (1966) 135-145.

7. J.A. Goleb, Uranium isotope investigations by atomic absorption, Anal. Chem. 35 (1963) 1978.

8. H. Liu, A. Quentmeier, K. Niemax, Diode laser absorption measurement of uranium isotope ratios in solid samples using laser ablation, Spectrochim. Acta Part B 57 (2002) 1611-1623.

9. L.E. Burkhart, G. Stukenbroeker, S. Adams, Isotope shifts in uranium spectra, Phys. Rev. 75 (1949) 83-85.

10. M.K. Mahani, A.R. Khanchi, M. Heidari, A. Ahmadi, A novel inductively coupled plasma atomic emission spectrometry method for uranium isotope ratio measurements using chemometric techniques, J. Anal. At. Spectrom. 25 (2010) 1659-1660.

11. P.S. Goodall, S.G. Johnson, Isotopic uranium determination by inductively coupled plasma atomic emission spectrometry using conventional and laser ablation sample introduction, J. Anal. At. Spectrom. 11 (1996) 57-60.

12. M.C. Edelson, V.A. Fassel, Isotopic abundance determinations by inductively coupled plasma atomic emission spectroscopy, Anal. Chem. 53 (1981) 2345-2347. 
13. P. Goodall, S.G. Johnson, High-resolution inductively coupled plasma atomic emission spectrometry for the determination of burnup in spent nuclear fuel, Appl. Spectrosc. 51 (1997) 182-187.

14. M. Krachler, D.H. Wegen, Promises and pitfalls in the reliable determination of U-233 using high resolution ICP-OES, J. Anal. At. Spectrom. 27 (2012) 335-339.

15. M. Krachler, P. Carbol, Validation of isotopic analysis of depleted, natural and enriched uranium using high resolution ICP-OES, J. Anal. At. Spectrom. 26 (2011) 293-299.

16. W. Pietsch, A. Petit, A. Briand, Isotope ratio determination of uranium by optical emission spectroscopy on a laser-produced plasma - basic investigations and analytical results, Spectrochim. Acta Part B 53 (1998) 751-761.

17. D.A. Cremers, A. Beddingfield, R. Smithwick, R.C. Chinni, C.R. Jones, B. Beardsley, L. Karch, Monitoring uranium, hydrogen, and lithium and their isotopes using a compact laserinduced breakdown spectroscopy (LIBS) probe and high-resolution spectrometer, Appl. Spectrosc. 66 (2012) 250-261.

18. B.W. Smith, A. Quentmeier, M. Bolshov, K. Niemax, Measurement of uranium isotope ratios in solid samples using laser ablation and diode laser-excited atomic fluorescence spectrometry, Spectrochim. Acta Part B 54 (1999) 943-958.

19. G.M. Murray, S.J. Weeks, M.C. Edelson, Determination of uranium isotopes in a complex matrix by optical spectroscopy, J. Alloys Compd. 181 (1992) 57-62.

20. R.W. Shaw, C.M. Barshick, L.W. Jennings, J.P. Young, J.M. Ramsey, Discharge conditioning for isotope ratio measurements by glow discharge optogalvanic spectroscopy, Rapid Commun. Mass Spectrom. 10 (1996) 316-320.

21. P. Pianarosa, Y. Demers, J.M. Gagné, Isotopic analysis by optogalvanic spectroscopy, J. Opt. Soc. Am. B 1 (1984) 704-709.

22. M. Krachler, R. Alvarez-Sarandes, S. Van Winckel, Challenges in the quality assurance of elemental and isotopic analyses in the nuclear domain benefitting from high resolution ICPOES and sector field ICP-MS, J. Radioanal. Nucl. Chem. 304 (2015) 1201-1209.

23. G.M. Hieftje, Introduction - A forward-looking perspective, in Inductively coupled plasma spectrometry and its applications, $2^{\text {nd }}$ ed., Blackwell Publishing Ltd, (2006) pp. 1-26. 
24. J.W. Olesik, Inductively coupled plasma mass spectrometry, in Inorganic mass spectrometry - fundamentals and applications, $1^{\text {st. }}$ ed., Marcel Dekker, New York, NY, USA (2000) pp. $67-158$.

25. C.M. Davies, H.H. Telle, D.J. Montgomery, R.E. Corbett, Quantitative analysis using remote laser-induced breakdown spectroscopy (LIBS), Spectrochim. Acta Part B 50 (1995) 1059-1075.

26. S.K. Sharma, A.K. Misra, P.G. Lucey, R.C. Wiens, S.M. Clegg, Combined remote LIBS and raman spectroscopy at $8.6 \mathrm{~m}$ of sulfur-containing minerals, and minerals coated with hematite or covered with basaltic dust, Spectrochim. Acta Part A 68 (2007) 1036-1045.

27. H. Hou, G.C.Y. Chan, X. Mao, R. Zheng, V. Zorba, R.E. Russo, Femtosecond filament-laser ablation molecular isotopic spectrometry, Spectrochim. Acta Part B 113 (2015) 113-118.

28. G. Rossi, Isotopic analysis of uranium by an optical spectral method-IV: Study of the suitability of some uranium lines for the absolute determination of isotopic ratios, Spectrochim. Acta Part B 26 (1971) 271-278.

29. G. Rossi, M. Mol, Isotopic analysis of uranium by an optical spectral method-III: Determination of U235/U238 ratios with a hollow-cathode source and a direct-reading attachment, Spectrochim. Acta Part B 24 (1969) 389-398.

30. J.L. Saunderson, A method of uranium isotope analysis by direct reading emission spectroscopy, Talanta 6 (1960) 63-70.

31. J.A. Leys, R.E. Perkins, Uranium isotope analysis by optical emission spectrometry, Anal. Chem. 38 (1966) 1099-1101.

32. D.P. Baldwin, D.S. Zamzow, A.P. Dsilva, High-resolution spectroscopy using an acoustooptic tunable filter and a fiber-optic Fabry-Perot interferometer, Appl. Spectrosc. 50 (1996) 498-503.

33. E.J. Judge, J.E. Barefield, J.M. Berg, S.M. Clegg, G.J. Havrilla, V.M. Montoya, L.A. Le, L.N. Lopez, Laser-induced breakdown spectroscopy measurements of uranium and thorium powders and uranium ore, Spectrochim. Acta Part B 83-84 (2013) 28-36.

34. I. Choi, G.C.Y. Chan, X. Mao, D.L. Perry, R.E. Russo, Line selection and parameter optimization for trace analysis of uranium in glass matrices by laser-induced breakdown spectroscopy (LIBS), Appl. Spectrosc. 67 (2013) 1275-1284. 
35. C. Pasquini, J. Cortez, L.M.C. Silva, F.B. Gonzaga, Laser induced breakdown spectroscopy, J. Braz. Chem. Soc. 18 (2007) 463-512.

36. P. Lindblom, New compact echelle spectrographs with multichannel time-resolved recording capabilities, Anal. Chim. Acta 380 (1999) 353-361.

37. P. Robert, C. Fabre, J. Dubessy, M. Flin, M.-C. Boiron, Optimization of micro-laser induced breakdown spectroscopy analysis and signal processing, Spectrochim. Acta Part B 63 (2008) 1109-1116.

38. G.C.Y. Chan, X. Mao, I. Choi, A. Sarkar, O.P. Lam, D.K. Shuh, R.E. Russo, Multiple emission line analysis for improved isotopic determination of uranium - a computer simulation study, Spectrochim. Acta Part B 89 (2013) 40-49.

39. R.C. Chinni, D.A. Cremers, L.J. Radziemski, M. Bostian, C. Navarro-Northrup, Detection of uranium using laser-induced breakdown spectroscopy, Appl. Spectrosc. 63 (2009) 12381250.

40. C.A. Smith, M.A. Martinez, D.K. Veirs, D.A. Cremers, Pu-239/Pu-240 isotope ratios determined using high resolution emission spectroscopy in a laser-induced plasma, Spectrochim. Acta Part B 57 (2002) 929-937.

41. K. Song, H. Cha, J. Lee, Y.I. Lee, Investigation of the line-broadening mechanism for laserinduced copper plasma by time-resolved laser-induced breakdown spectroscopy, Microchem. J. 63 (1999) 53-60.

42. S. Merk, A. Demidov, D. Shelby, I.B. Gornushkin, U. Panne, B.W. Smith, N. Omenetto, Diagnostic of laser-induced plasma using abel inversion and radiation modeling, Appl. Spectrosc. 67 (2013) 851-859.

43. P. Yan, W. Luo, J. Zhang, L. Wang, Measurements of hyperfine structure and isotope shift for atomic uranium by laser induced-fluorescence spectroscopy, Chin. J. Lasers 1 (1992) 409-413.

44. L.J. Radziemski, S. Gerstenkorn, P. Luc, Uranium transitions and energy-levels which may be useful in atomic-photo-ionization schemes for separating U-238 and U-235, Opt. Comm. 15 (1975) 273-276.

45. B.A. Palmer, R.J. Engleman, R.A. Keller, An atlas of uranium emission intensities in a hollow-cathode discharge, LA-8251-MS, Los Alamos National Laboratory, (1980). 
46. J. Blaise, L.J. Radziemski, Energy-levels of neutral atomic uranium (U I), J. Opt. Soc. Am. 66 (1976) 644-659.

47. J. Blaise, J.F. Wyart, J. Verges, R. Engleman, B.A. Palmer, L.J. Radziemski, Energy-levels and isotope shifts for singly ionized uranium (U II), J. Opt. Soc. Am. B 11 (1994) 18971929.

48. W.J. Childs, O. Poulsen, L.S. Goodman, High-precision measurement of 235U ground-state hyperfine structure of laser-rf double resonance, Opt. Lett. 4 (1979) 35-37.

49. U. Nielsen, P. Thorsen, O. Poulsen, H. Crosswhite, 235U II hyperfine structures measured by collinear fast-beam-laser and radio-frequency-laser double-resonance spectroscopy, J. Opt. Soc. Am. B 1 (1984) 284-292.

50. G.N. Rao, M.N. Reddy, E. Hecht, Atomic hyperfine structure studies using temperature/current tuning of diode lasers: An undergraduate experiment, Am. J. Phys. 66 (1998) 702-712.

51. P. Jacquinot, R. Klapisch, Hyperfine spectroscopy of radioactive atoms, Rep. Prog. Phys. 42 (1979) 773-832.

52. Gerstenk.S, P. Luc, Bauchear.C, D. Merle, Hyperfine-structure and nuclear and quadrupolemoments of 235 isotope of uranium, Journal De Physique 34 (1973) 805-812.

53. O. Axner, J. Gustafsson, N. Omenetto, J.D. Winefordner, Line strengths, A-factors and absorption cross-sections for fine structure lines in multiplets and hyperfine structure components in lines in atomic spectrometry - a user's guide, Spectrochim. Acta Part B 59 (2004) 1-39.

54. J.M. Gagne, J.P. Saintdizier, P. Pianarosa, Odd-even staggering of U-235 from $5027 \AA$ Aine in U I, Opt. Comm. 20 (1977) 269-270.

55. R. Engleman, B.A. Palmer, Precision isotope shifts for the heavy-elements .1. Neutral uranium in the visible and near-infrared, J. Opt. Soc. Am. 70 (1980) 308-317. 
Table 1 Constraints in the five spectral-decomposition algorithms.

\begin{tabular}{cl}
\hline Algorithm & \multicolumn{1}{c}{ Constraints } \\
\hline \hline I & No constraint; $\lambda_{235}^{o}, \lambda_{238}^{o}, \omega_{235}$, and $\omega_{238}$ are all independent fitting variables \\
II & $\lambda_{238}^{o}-\lambda_{235}^{o}=$ published isotopic shift \\
III & $\lambda_{238}^{o}-\lambda_{235}^{o}=$ published isotopic shift, and $\omega_{235}=\omega_{238}$ \\
IV & $\lambda_{238}^{o}-\lambda_{235}^{o}=$ published isotopic shift, and $\omega_{235}=k \omega_{238}$ \\
V & $\begin{array}{l}\text { Each hyperfine component of }{ }^{235} \mathrm{U} \text { is fitted with } \omega_{235, H F S-i}=\omega_{238}, \text { and } \lambda_{238}^{o}- \\
\end{array} \quad \lambda_{235, H F S-i}^{o}=$ published isotopic and hyperfine shifts \\
\hline
\end{tabular}


Table 2 Spectroscopic constant, isotopic shift (IS) and hyperfine constants for ${ }^{235} \mathrm{U}$ for U I 682.691 $\mathrm{nm}$ and U II $424.437 \mathrm{~nm}$ lines [43, 45-49]. Emission wavelengths and energy levels for $682.691 \mathrm{~nm}$ and $424.437 \mathrm{~nm}$ lines refer to emission from ${ }^{238} \mathrm{U}$. Isotopic shifts for ${ }^{235} \mathrm{U}$ refer to the energy shifts of the centroid of the hyperfine patterns, relative to ${ }^{238} \mathrm{U}$. Isotopic shift and hyperfine constants are given in unit of milli-kayser $\left(1 \mathrm{mK}=0.001 \mathrm{~cm}^{-1}\right)$.

\begin{tabular}{lccccc}
\hline & Energy / eV & Term & IS / mK & $A / \mathrm{mK}$ & $B / \mathrm{mK}$ \\
\hline \hline U I 682.691 nm & & & & \\
Upper level & $1.81556[45]$ & ${ }^{7} \mathrm{M}_{6}[46]$ & $-380[46]$ & $-4.03[43]$ & $+283.2[43]$ \\
Lower level & $0[45]$ & ${ }^{5} \mathrm{~L}_{6}^{\circ}[46]$ & $0[46]$ & $-2.02[48]$ & $+136.9[48]$ \\
& & & & & \\
U II 424.437 nm & & & & & \\
Upper level & $2.92025[45]$ & $\boldsymbol{J}=5.5^{\dagger}[47]$ & $-1394.5^{*}[47]$ & $-3.899[49]$ & $-26.59[49]$ \\
Lower level & $0[45]$ & ${ }^{4} \mathrm{I}_{4.5}^{\circ}[47]$ & $0[47]$ & $-2.565[49]$ & $+33.92[49]$ \\
\hline
\end{tabular}

${ }^{*}$ IS was converted from ${ }^{234} \mathrm{U}-{ }^{238} \mathrm{U}$ to ${ }^{235} \mathrm{U}-{ }^{238} \mathrm{U}$ with a correction factor $1.194[38,54,55]$.

Term symbol is not documented for this energy level as it is a mixed level $\left(f^{3} s p+f^{3} d p\right)$ [47]. 
Table $3{ }^{235} \mathrm{U}$ hyperfine structure for the U I $682.691 \mathrm{~nm}$ emission line. The three entries listed for each hyperfine transition refer to the shift of transition energy (top, in unit of $\mathrm{mK}$ with respective to the centroid of the hyperfine pattern), fractional transition strength (middle), and air emission wavelength (bottom, in unit of $\mathrm{nm}$ ). The total angular momentums of the upper and lower levels are denoted by $\boldsymbol{F}^{\prime}$ and $\boldsymbol{F}^{\prime \prime}$, respectively. The $682.691 \mathrm{~nm}$ emission wavelength refers to ${ }^{238} \mathrm{U}$, and the centroid of the ${ }^{235} \mathrm{U}$ hyperfine pattern shifts from the ${ }^{238} \mathrm{U}$ emission by $-380 \mathrm{mK}$ [46].

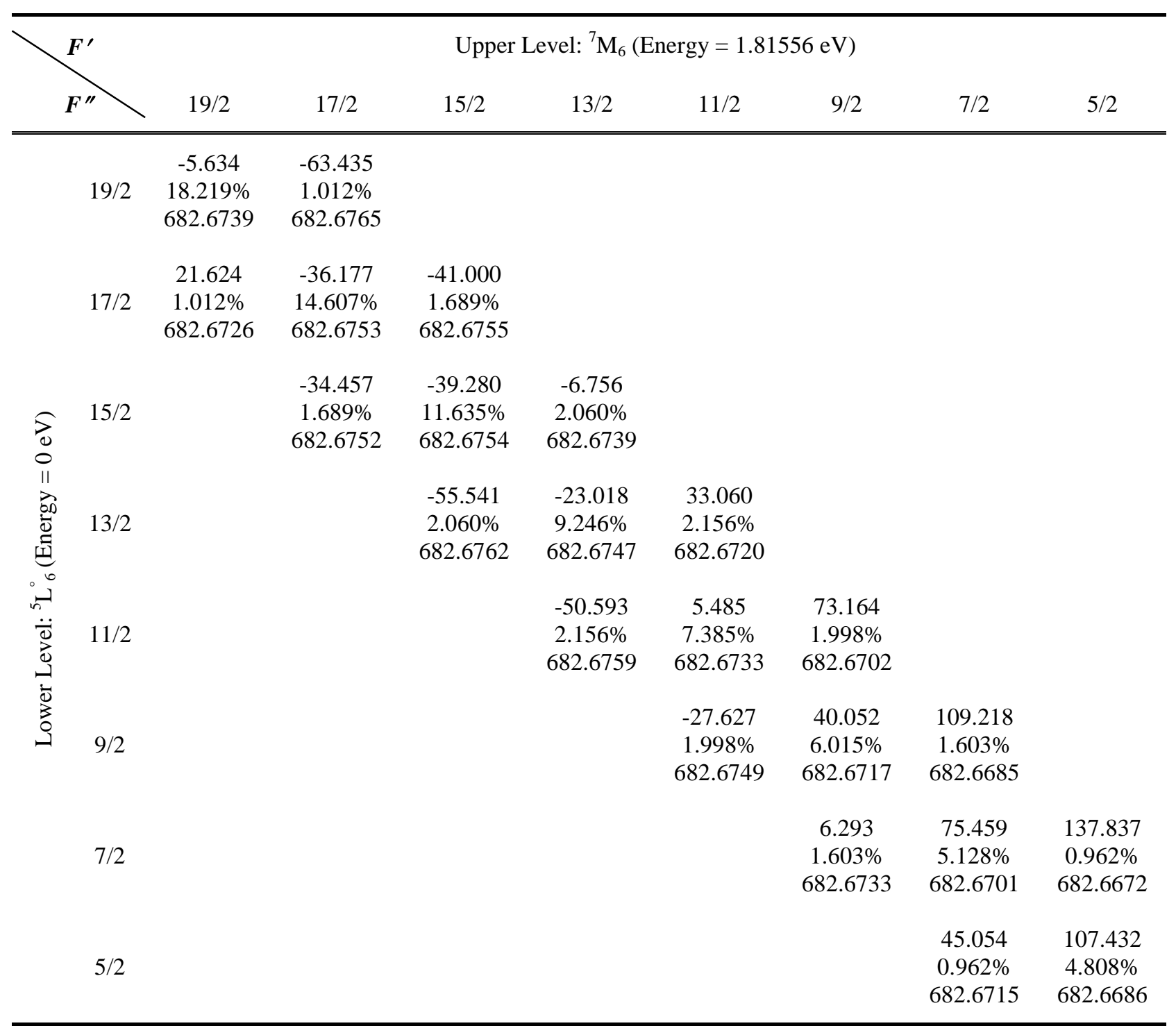


Table $4{ }^{235} \mathrm{U}$ hyperfine structure for the U II $424.437 \mathrm{~nm}$ emission line. The three entries listed for each hyperfine transition refer to the shift of transition energy (top, in unit of $\mathrm{mK}$ with respective to the centroid of the hyperfine pattern), fractional transition strength (middle), and air emission wavelength (bottom, in unit of nm). The total angular momentums of the upper and lower levels are denoted by $\boldsymbol{F}^{\prime}$ and $\boldsymbol{F}^{\prime \prime}$, respectively. The $424.437 \mathrm{~nm}$ emission wavelength refers to ${ }^{238} \mathrm{U}$, and the centroid of the ${ }^{235} \mathrm{U}$ hyperfine pattern shifts from the ${ }^{238} \mathrm{U}$ emission by $-1394.5 \mathrm{mK}$ [47]. Term symbol for the upper energy level is undocumented as it is a mixed level $\left(f^{3} s p+f^{3} d p\right)$ [47].

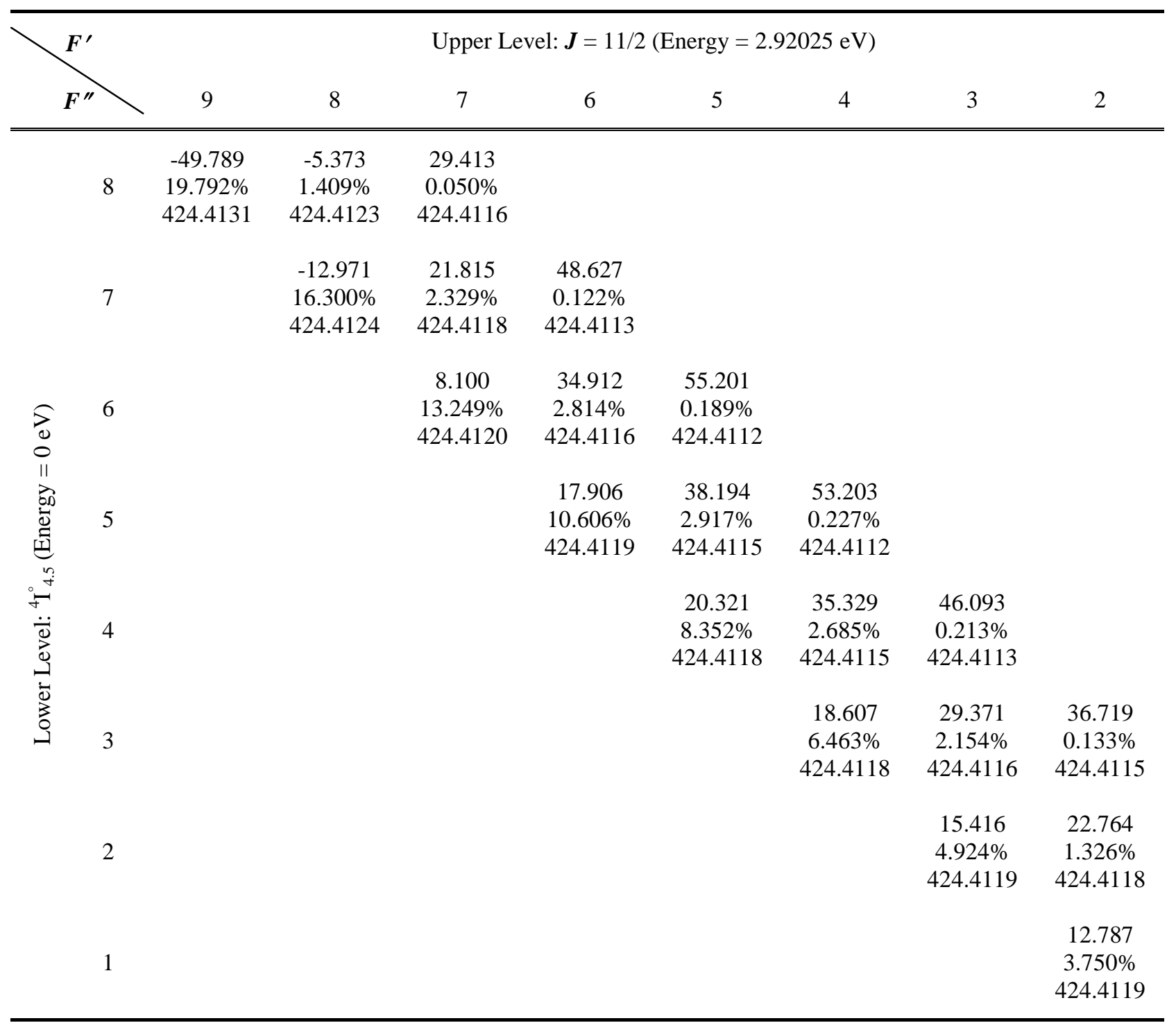




\section{Figure Captions}

Figure 1 Experimental LIBS spectra from blank soil (NIST SRM 2710a) and soil sample doped with $1.1 \% \mathrm{w} / \mathrm{w}$ uranium measured at (a) U I $682.691 \mathrm{~nm}$, and (b) U II $424.437 \mathrm{~nm}$. The doped uranium was ${ }^{235} \mathrm{U}$ enriched with an abundance of $64.37 \%$. Two decomposed spectral profiles, correspond to ${ }^{235} \mathrm{U}$ and ${ }^{238} \mathrm{U}$, are also shown under the experimental uranium spectral profiles to illustrate the fitting parameters in the decomposition algorithms.

Figure 2 (a) Simulated emission profiles from a $50 \%-50 \%{ }^{235} \mathrm{U}-{ }^{238} \mathrm{U}$ sample for U I $682.691 \mathrm{~nm}$. The simulation assumes that each ${ }^{235} \mathrm{U}$ HFS component and the ${ }^{238} \mathrm{U}$ profile are Lorentzian in shape with a 20-pm width. Also included in the figure are the eight principal HFS components (in this case, $\Delta F=F^{\prime}-F^{\prime \prime}=0$ ). (b) Fitted peak width ratios for the ${ }^{235} \mathrm{U}$ to ${ }^{238} \mathrm{U}$ isotopic peak as a function of the ${ }^{238} \mathrm{U}$ line width. The ratios can be well represented as a sum of two exponential decays (the solid line) and define the value of $\boldsymbol{k}$ in Algorithm IV for spectral decomposition.

Figure 3 (a) Simulated emission profiles from a $50 \%-50 \%{ }^{235} \mathrm{U}-{ }^{238} \mathrm{U}$ sample for U II $424.437 \mathrm{~nm}$. The simulation assumes that each ${ }^{235} \mathrm{U}$ HFS component and the ${ }^{238} \mathrm{U}$ profile are Lorentzian in shape with a 10-pm width. Also included in the figure are the eight principal HFS components (in this case, $\Delta F=F^{\prime}-F^{\prime \prime}=+1$ ). (b) Fitted peak width ratios for the ${ }^{235} \mathrm{U}$ to ${ }^{238} \mathrm{U}$ isotopic peak as a function of the ${ }^{238} \mathrm{U}$ line width. The ratios can be well represented as a sum of two exponential decays (the solid line) and define the value of $\boldsymbol{k}$ in Algorithm IV for spectral decomposition.

Figure 4 The determined isotopic ratios of ${ }^{235} U /\left({ }^{235} U+{ }^{238} U\right)$ from the use of different algorithms for spectral decomposition of the two $U$ isotopic peaks measured at 
(a) U I $682.691 \mathrm{~nm}$, and (b) U II $424.437 \mathrm{~nm}$. The error bars represent standard deviations of six replicated measurements. The dashed line represents the certified ${ }^{235} \mathrm{U}$ ratio $(64.37 \%)$

Figure 5 Calibration curves showing total $U$ emission (sum of decomposed ${ }^{235} \mathrm{U}$ and ${ }^{238} \mathrm{U}$ signals) as a function of uranium concentrations in soil for emission lines (a) U I $682.691 \mathrm{~nm}$, and (b) U II $424.437 \mathrm{~nm}$. The error bars represent standard deviations of six replicated measurements. The straight lines represent the calibration curves obtained with uncertainty-weighted linear regression. 
Figure 1a

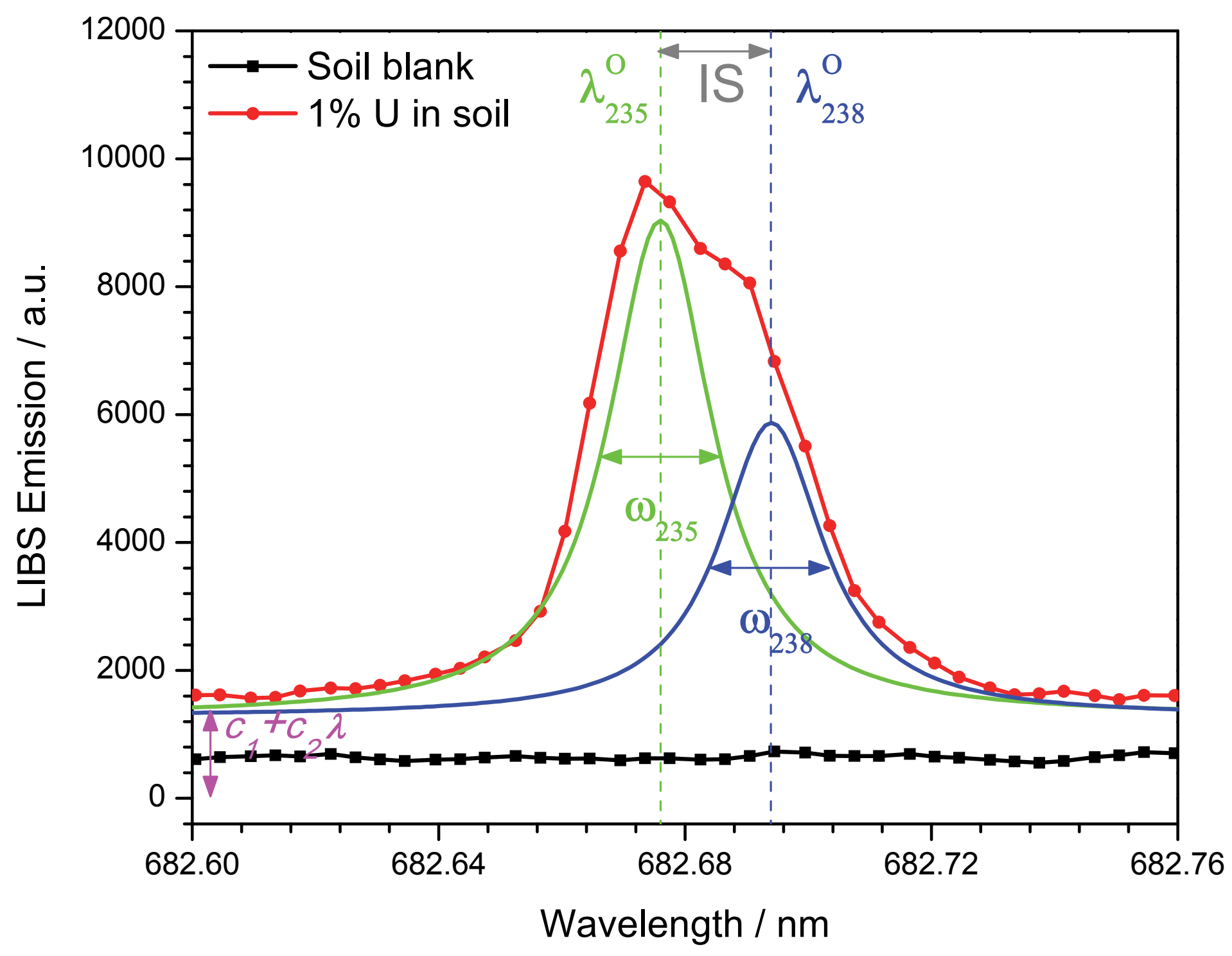


Figure $1 b$

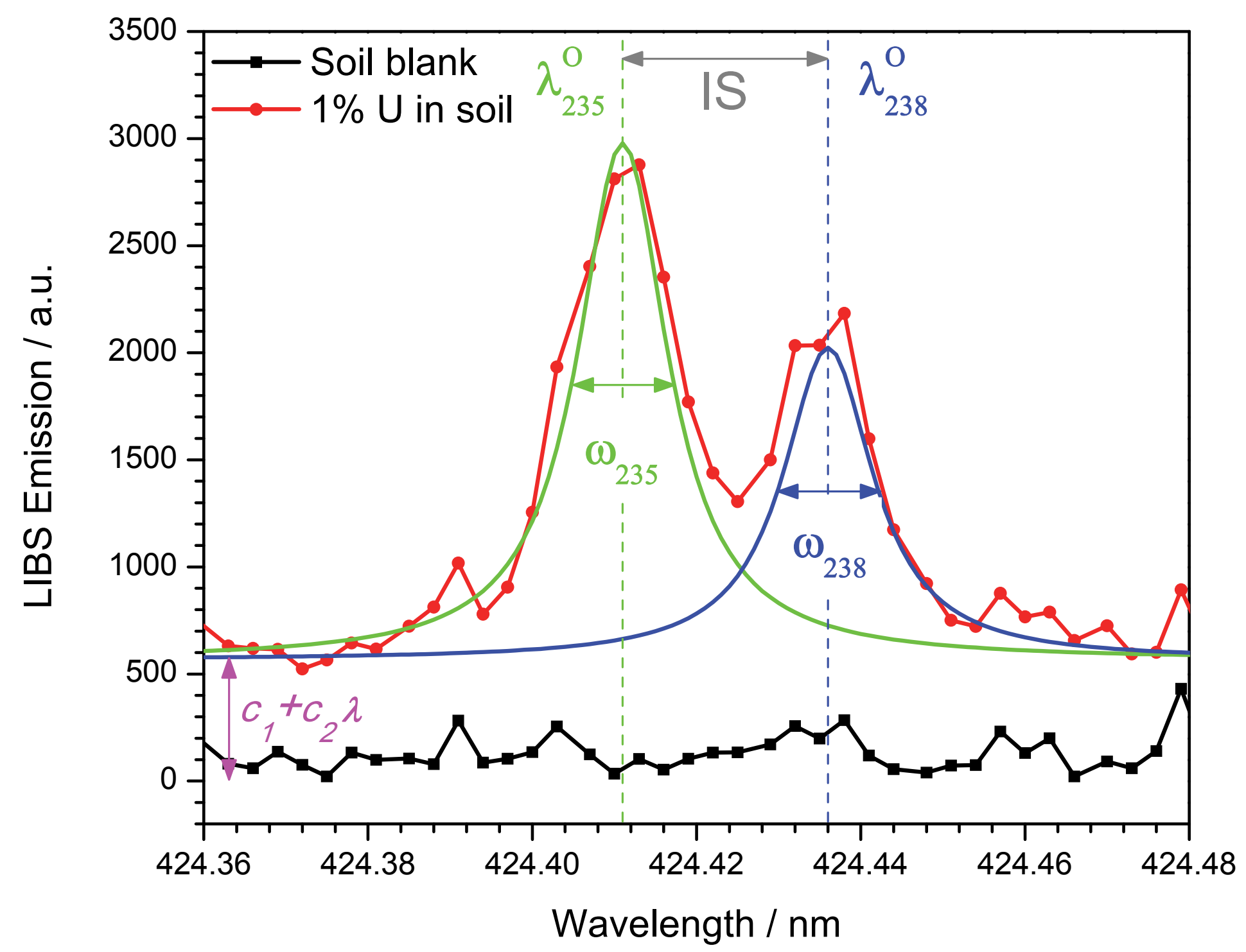


Figure 2a

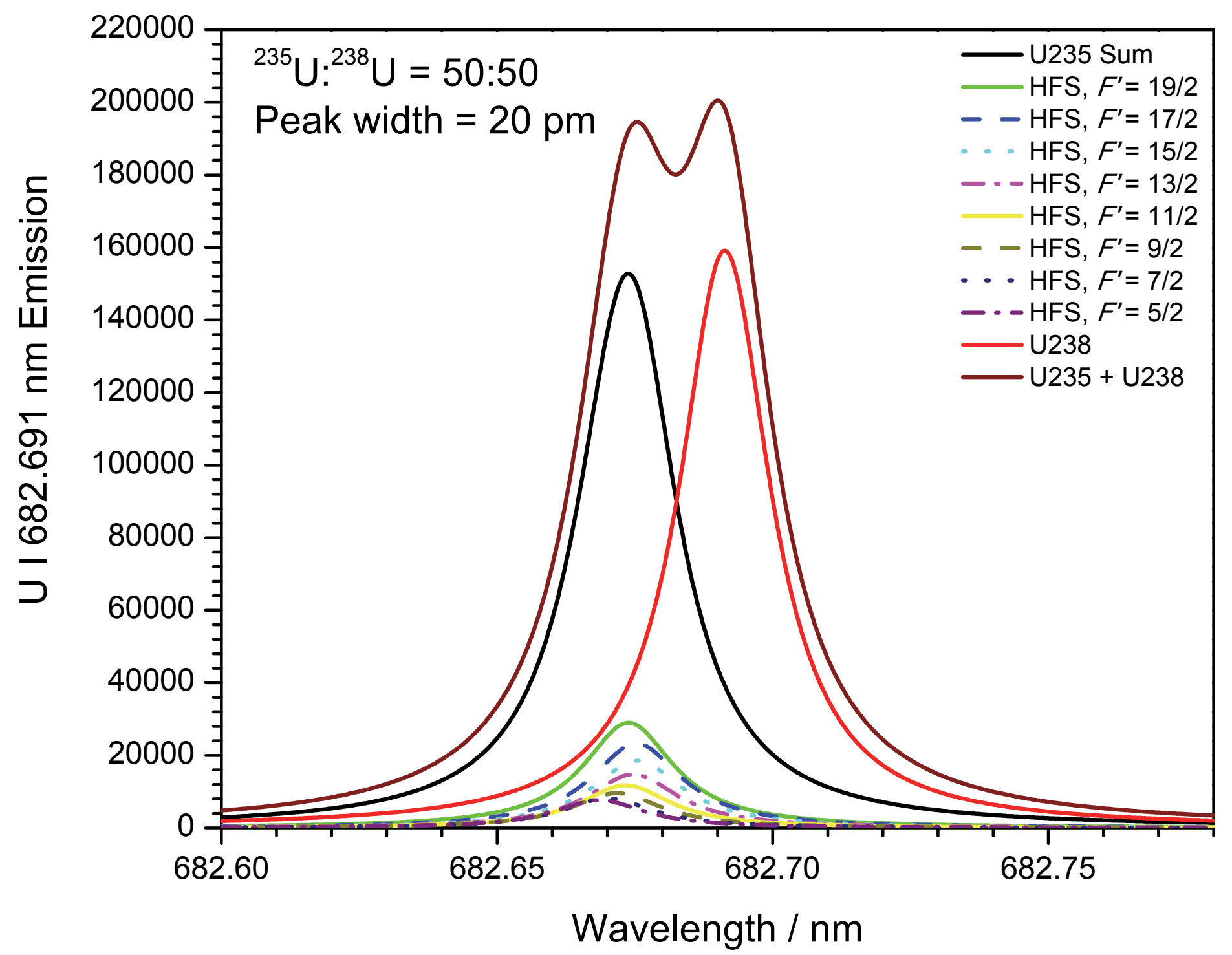


Figure 2b

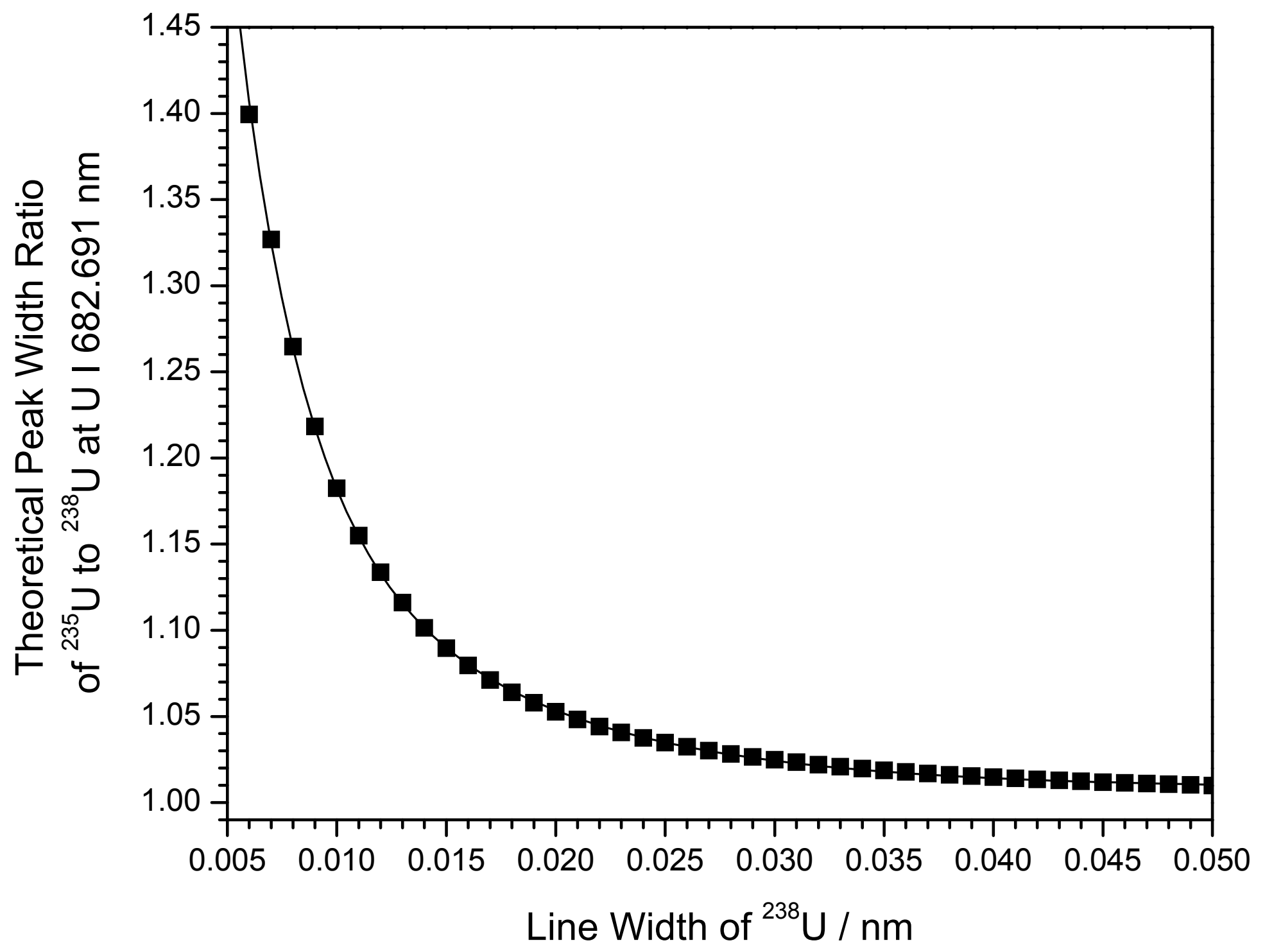


Figure $3 \mathrm{a}$

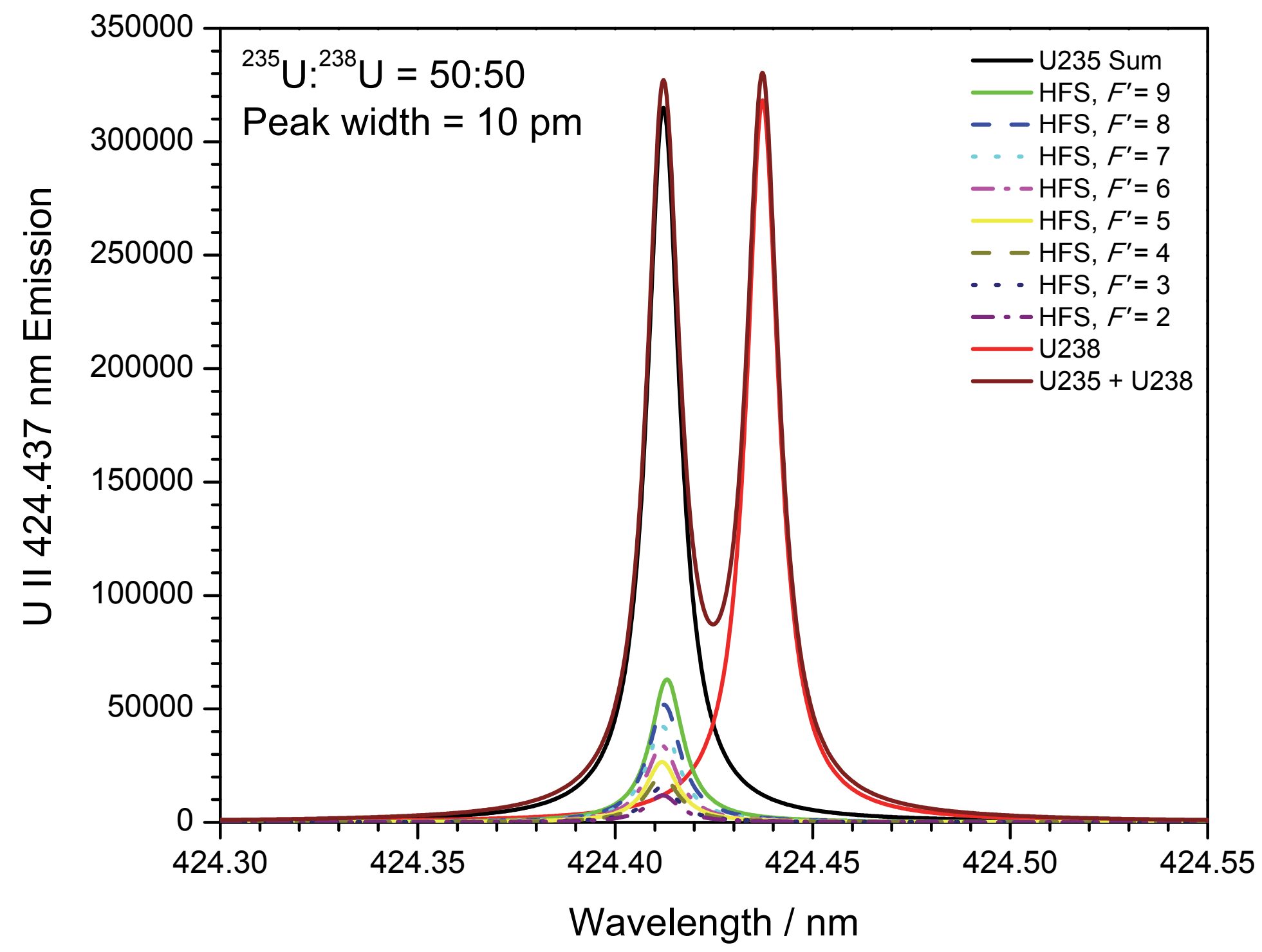


Figure $3 b$

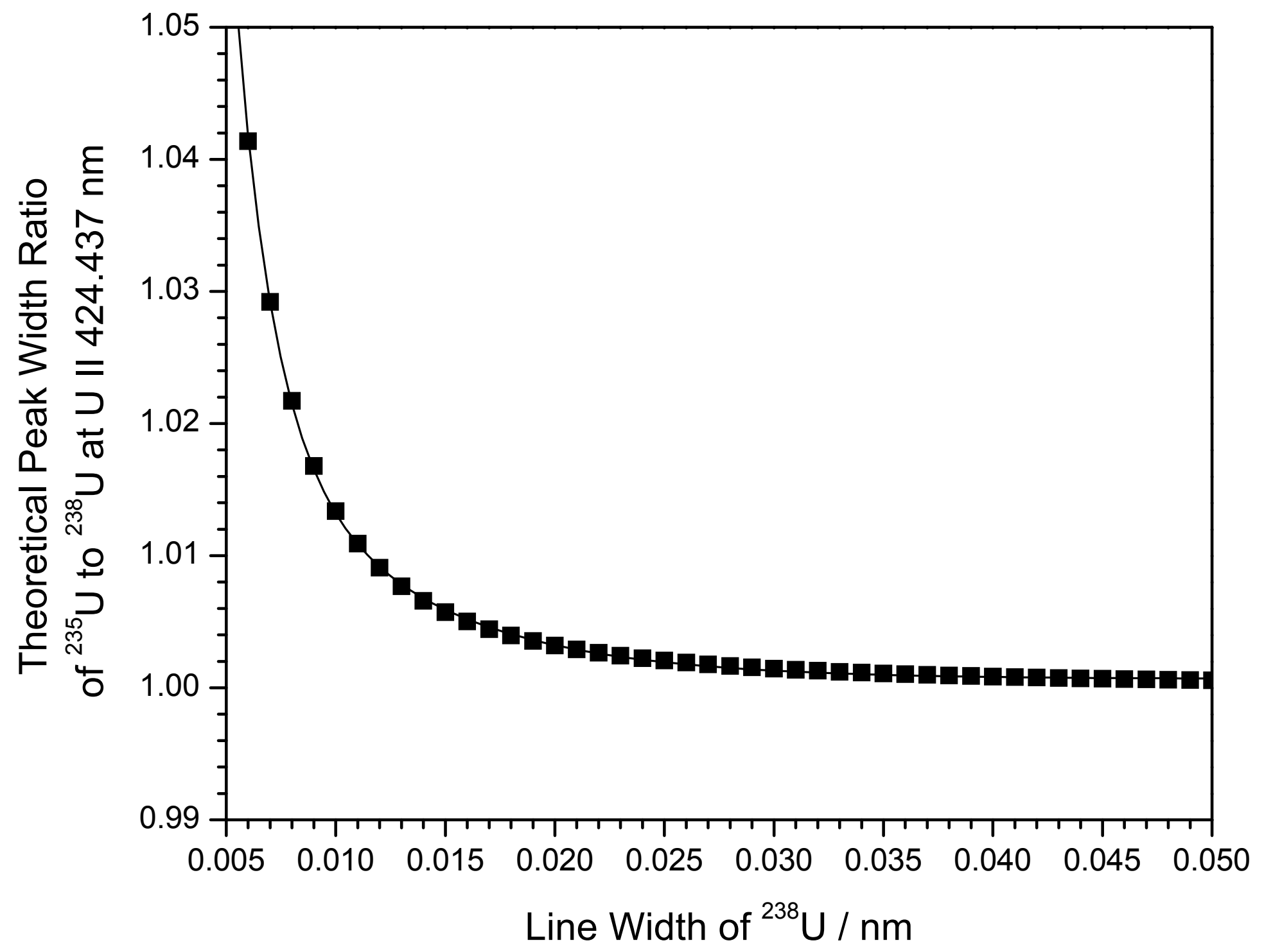


Figure 4a

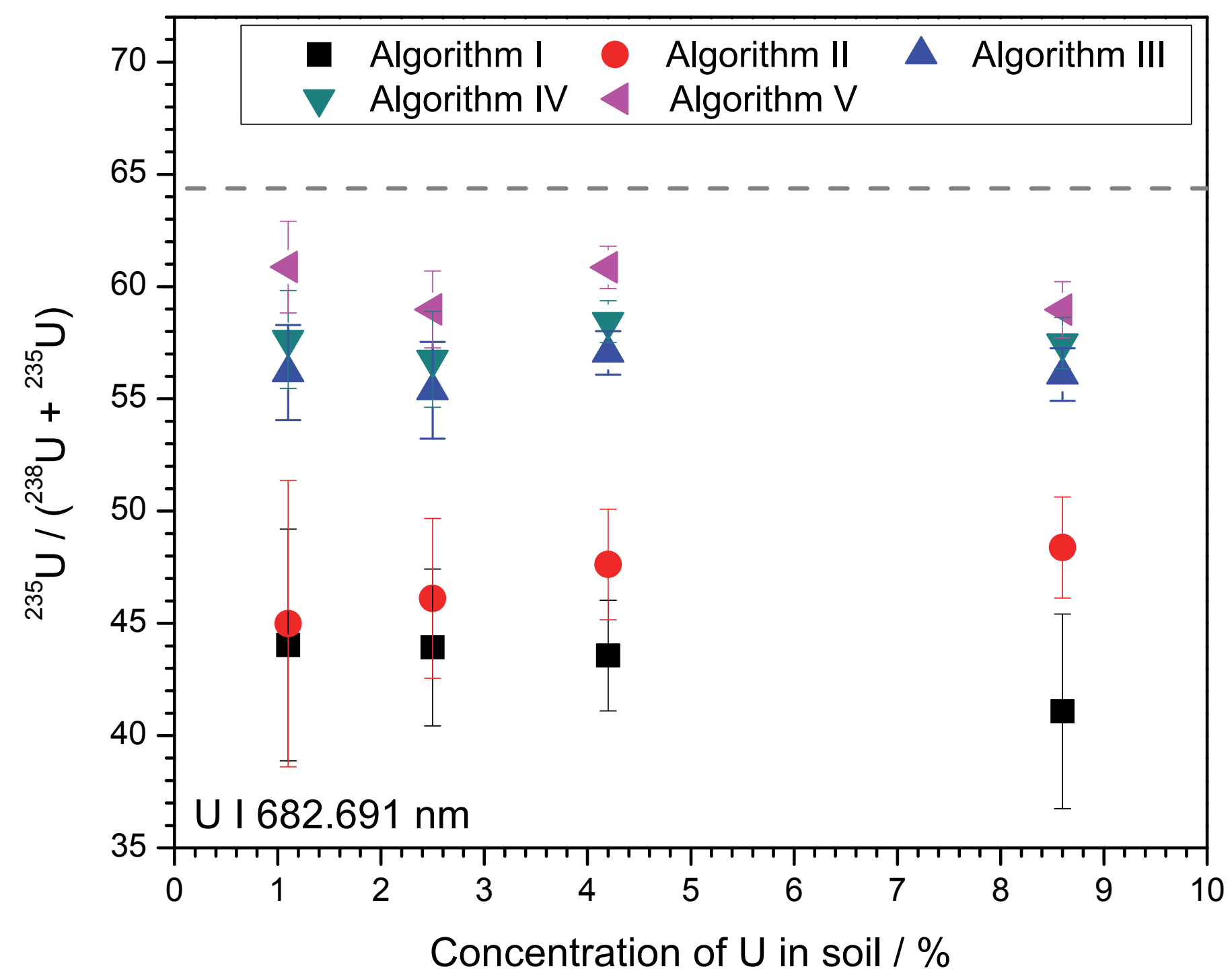


Figure $4 b$

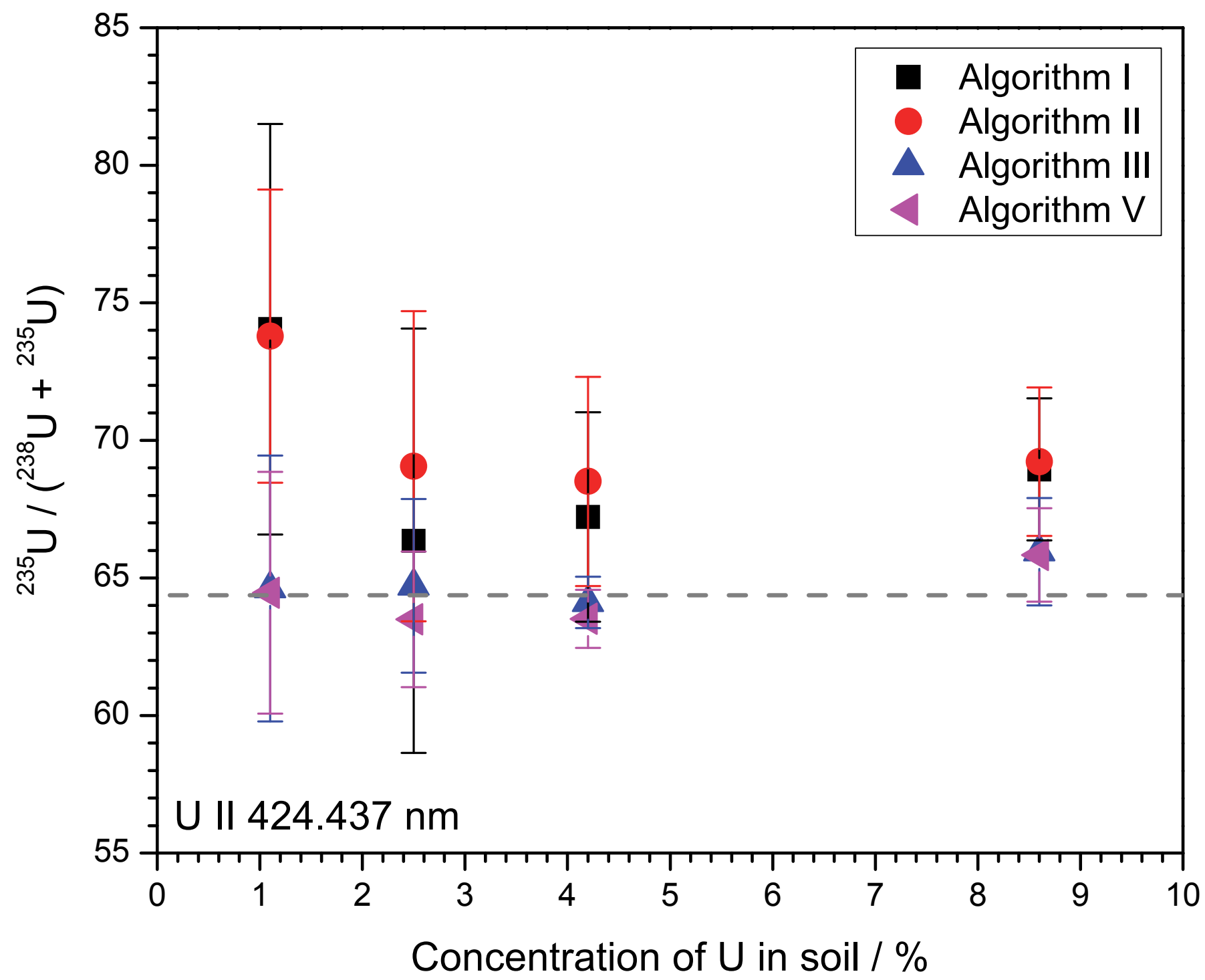


Figure 5a

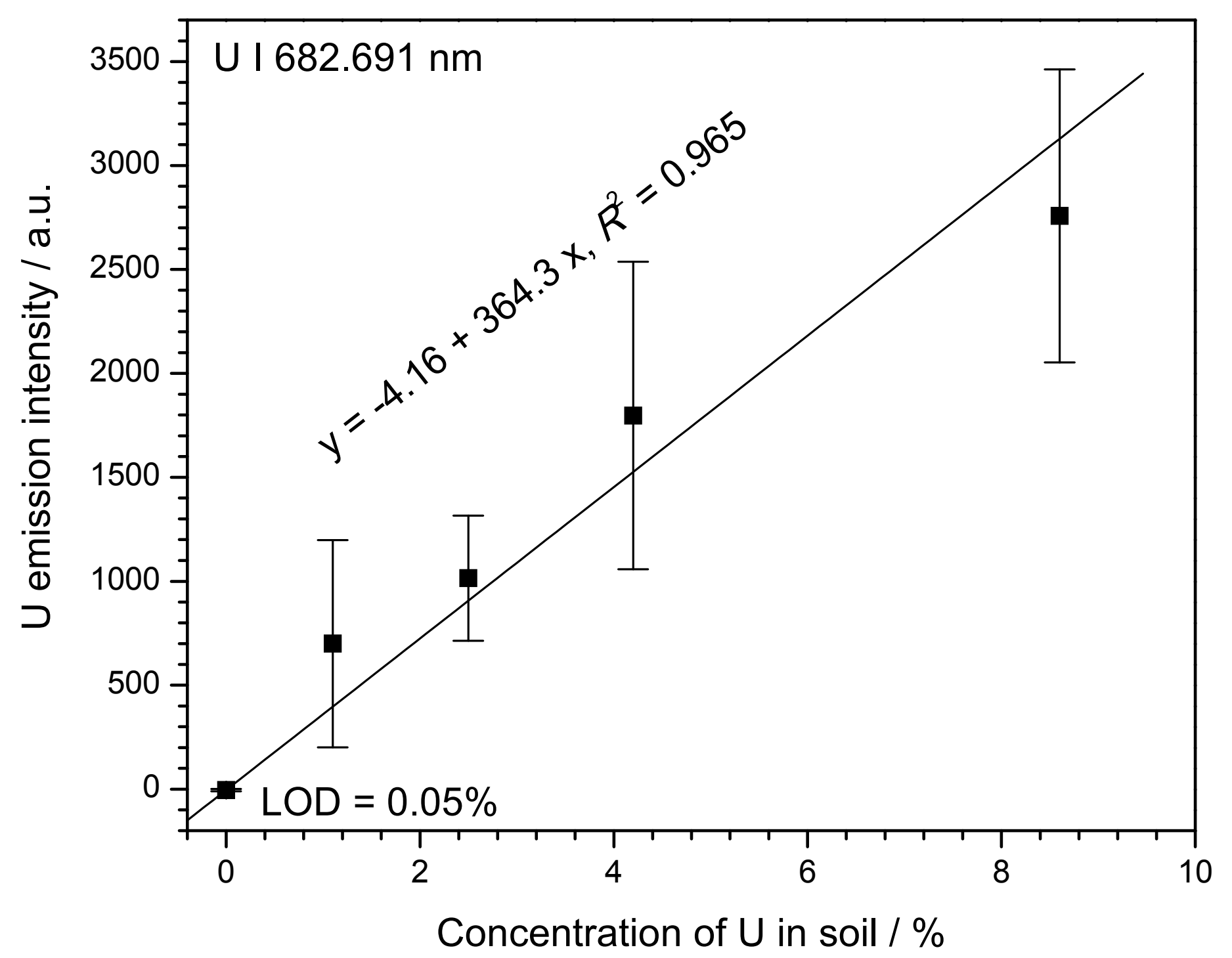


Figure $5 b$

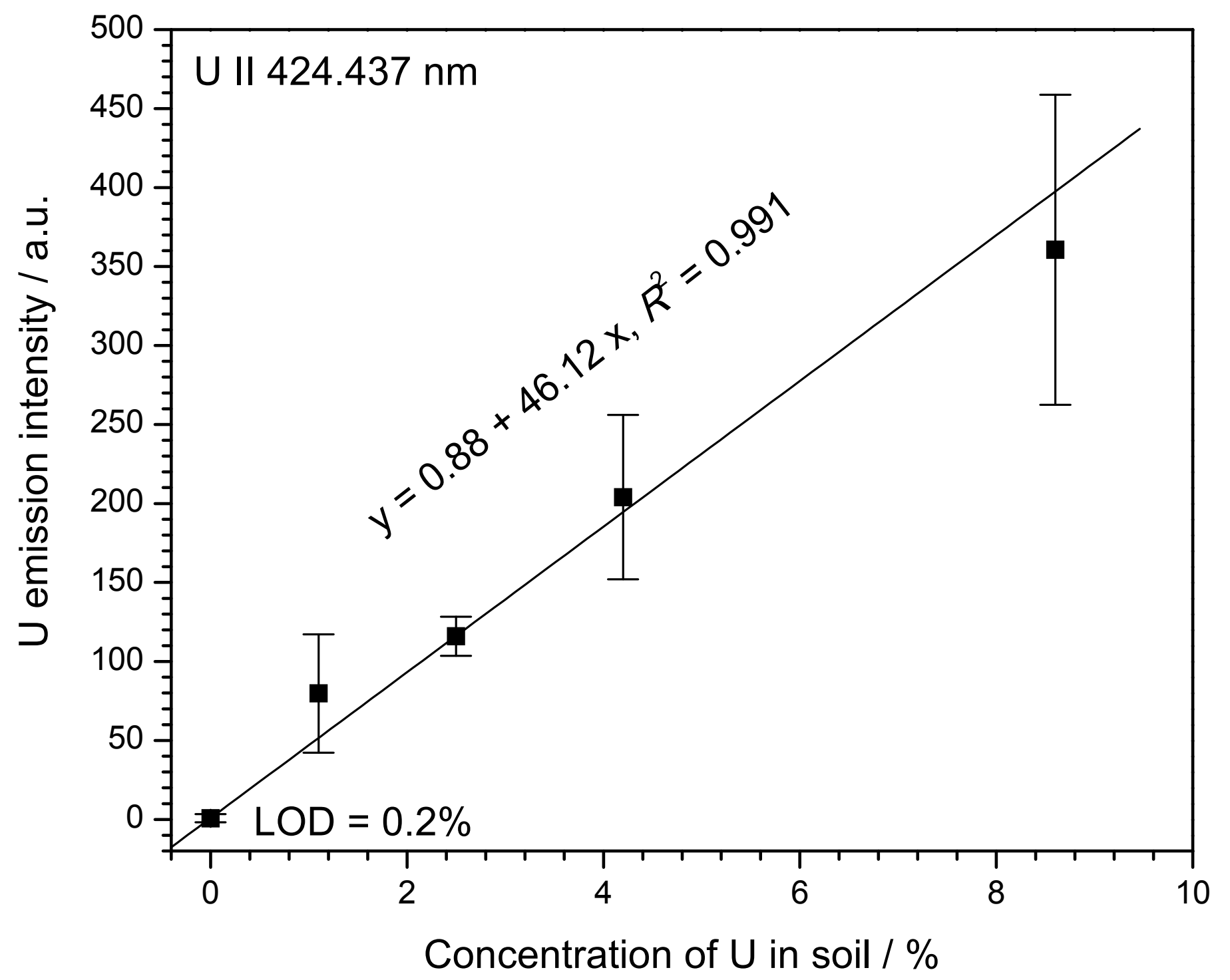

